\title{
An Innovative Approach to Investigate the Effects of Artificial Intelligence Based on Complex Bipolar Picture Fuzzy Information
}

\author{
Naeem Jan, ${ }^{1}$ Bushra Akram, ${ }^{1}$ Abdul Nasir ${ }^{D},{ }^{1}$ Mohsin S. Alhilal, ${ }^{2}$ Amerah Alabrah ${ }^{1}{ }^{2}$ \\ and Naziha Al-Aidroos $\mathbb{D D}^{3}$ \\ ${ }^{1}$ Department of Mathematics, Institute of Numerical Science, Gomal University, Dera Ismail Khan 29050, KPK, Pakistan \\ ${ }^{2}$ Department of Information Systems, College of Computer and Information Sciences, King Saud University, \\ Riyadh 11543, Saudi Arabia \\ ${ }^{3}$ Computer Science Department College of Computers and Information Technology, Hadhramout University, \\ Hadhramout, Yemen \\ Correspondence should be addressed to Naziha Al-Aidroos; naz.moh@hu.edu.ye
}

Received 24 November 2021; Revised 27 December 2021; Accepted 5 January 2022; Published 17 February 2022

Academic Editor: Sikandar Ali

Copyright (c) 2022 Naeem Jan et al. This is an open access article distributed under the Creative Commons Attribution License, which permits unrestricted use, distribution, and reproduction in any medium, provided the original work is properly cited.

\begin{abstract}
Artificial intelligence (AI) has made the life more efficient and powered many programs and services. AI is progressing rapidly, and the future is arriving faster than the predictions. Soon, AI will be more proficient as compared to humans in all aspects. Many industries are using AI for the analysis of data to find the best methods for investments. In this article, we developed the impacts of AI on different industries through the new concepts of complex bipolar picture fuzzy set (CBPFS) proposed in the current study. The CBPFS has an extensive structure that includes membership, abstinence, and nonmembership degrees with the ability to deal with multivariable problems. These degrees are fuzzy numbers between 0 and 1 inclusive; 0 being the lowest and 1 being the highest value for each degree, which reflect different meaning for membership, nonmembership, and abstinence. Furthermore, the paper explains the Cartesian product between CBPFSs and complex bipolar picture fuzzy relation (CBPFR) and its types with suitable example. Furthermore, through a comparison test with preexisting fuzzy set frameworks, some benefits of CBPFS are presented in this article.
\end{abstract}

\section{Introduction}

Uncertainty could be a common characteristic of each day decisions. When there are multiple possible outcomes from taking any course of decisions, a situation of uncertainty occurs. Human decisions are generally ambiguous and unpredictable. It will always be a part of our living. The difficulty in mathematics is determining way to characterize ambiguous groups of things. Meanwhile, Zadeh [1] introduced the idea of fuzzy sets (FSs) in 1965, which deal with the uncertainty. Fuzzy set assigns membership degree to each object in the set. The level of membership is a function that is ranging from $[0,1]$.

Klir and Folger [2] invented the crisp relations, which are used to investigate the interactions between crisp sets. The crisp relation only discussed just two possibilities yes or no.
Negoita and Ralescu [3] connected the FSs to system analysis. Laengla et al. [4] suggested a bibliometric evaluation of FSs. Xu et al. [5] optimized many goals stream shop scheduling using a genetic set of rules based on FSs, and Mewada et al. [6] expanded a fuzzy system with applications by extrapolating a fuzzy system. Adlassnig [7] employed fuzzy set in the field of medicine. Lu and Ruan [8] suggested an application of multiobjective group decision making. Gehrke et al. [9] commented on IVFSs. Mendel [10] presented the concepts of fuzzy relations (FRs) that are used to analyze the connections among the FSs. The FRs are indicating the degree of membership, whose values range from 0 to 1. Yu et al. [11] used FRs to assess uncertainty and provide some applications. Yeh and Bang [12] used the FRs to clustering analysis. Elkano et al. [13] employed aggregation functions for the composition of IVFRs. Atanassov et al. [14] 
proposed the novel concept of intuitionistic fuzzy set (IFS). Further, he defined the new idea of the interval valued intuitionistic fuzzy set (IVIFS). The IFS is an extensive form of FSs, whereas the IVIFS is an extensive form of IVFS. The IFSs discuss the membership and nonmembership degrees but FSs only discuss the membership degree. The membership and nonmembership degrees of IFSs must lie in the unit interval $[0,1]$, such that the sum of both the degrees is less than or equal to 1 . The IVIFS discusses the membership and nonmembership degrees in the form of intervals with some constraints. Szmidt and Kacprzyk [15] determined the distance between IFSs. De et al. [16] defined some IFSs operations. Burillo and Bustince [17] revealed the concepts of intuitionistic fuzzy relations (IFRs). It is a generalization of FRs. Deschrijver and Kerre [18] investigated the composition of IFRs. Cuong and Kreinovich [19] concocted a new idea of picture fuzzy set (PFSs) which is an extensive form of FSs and IFSs. Three stages of a component examined in PFSs, level of membership, level of abstinence, and level of nonmembership, with the sum of membership, abstinence, and nonmembership belong to $[0,1]$. Moreover, the concepts of picture fuzzy relation (PFRs) were also established. Ganie et al. [20] designed a few unused relationship coefficients of PFS and applications. Liu et al. [21] used the IVPFSs in decision making with some applications.

Later, Ramot et al. [22] developed the novel concept of complex fuzzy set (CFS), which expresses membership degree in the form of a complex number, i.e., $\phi_{c}(x)=\breve{a}_{c}(x)$ $e^{2 \pi \Phi_{\phi}(x) i}$, where $\breve{a}(\mathrm{x})$ is called amplitude term and $\Phi(x)$ is called phase term. Moreover, he also defined the complex fuzzy relation (CFR) which investigated the relationship between CFSs. Tamir et al. [23] gave an introduction of CFSs and CF logic together with their applications. Greenfield et al. [24] developed the new idea of interval valued complex fuzzy set (IVCFS), which changed the membership degree of a CFS from single number to an interval. Nasir et al. [25] used the IVCFRs in diagnosis and considered the life expectancy of patients. Ma et al. [26] proposed a strategy for multiple periodic factor prediction problems through CFSs. Alkouri et al. [27] developed the concepts of complex intuitionistic fuzzy set (CIFS). Garg and Rani [28] gave some results for measuring the CIFS. Jan et al. [29] derived the new concepts of complex intuitionistic fuzzy relation (CIFRs) with studying the relationship between CIFS. Nasir et al. [30] defined the new idea of interval valued complex intuitionistic fuzzy relation (IVCIFR) with application of cybersecurity. Akram et al. [31] invented the complex picture fuzzy set (CPFS). The complex picture fuzzy set is the more extended form of CFS and CIFS. The CPFS are discussed all of the three stages with the both amplitude and phase term. Nasir et al. [32] introduced the novel concepts of complex picture fuzzy relations (CPFRs) with application of network security. Zhang [33] presented the new ideas of bipolar fuzzy set (BFS) and bipolar fuzzy relations (BFRs). In a BFS, positive membership indicates what is certain to be possible, while negative membership indicates what is certain to be impossible or false. Lee [34] developed a new generalization of fuzzy sets known as bipolar valued fuzzy sets, in which the membership degree increased from $[0,1]$ to $[-1,1]$. In BFS, the degree of membership ' 0 ' indicates that elements are irrelevant to the corresponding property, membership degree $(0,1]$ indicates that some elements meet the corresponding property, and membership degree $[-1,0)$ indicates that some elements satisfy the implicit counter property. Mahmood and Hayat [35] investigated bipolar fuzzy and bipolar antifuzzy h-ideals on hemi-rings. Akram et al. [36] used the BFSs in planner graph and multigraphs and investigate the interesting structures to some properties. Alkouri et al. [37] introduced the new concepts of complex bipolar fuzzy set (CBFS). The structure of CBFS discussed the both amplitude and phase term. Ezhilmaran and Sankar [38] developed the concept of a bipolar intuitionistic fuzzy set (BIFS). The BIFS consists of the positive membership degree, negative membership degree, positive nonmembership degree, and negative nonmembership degree. Sindhu et al. [39] invented the novelty concepts of bipolar picture fuzzy set (BPFS). The BPFS are the generalization form of BFS and BIFS. The bipolar picture fuzzy sets (BPFS) also discuss the positive abstinence and negative abstinence. Nilsson [40] proposed the principles of artificial intelligence. Ramesh et al. [41] used the artificial intelligence which studies the application of medicine. Rondeau [42] discussed the AI with the application of wireless communication. Hirasawa et al. [43] used the AI in convolutional neural networks for detecting the gastric cancer. Anantrasirichai and Bull [44] investigated the AI in the creative industries. Yu et al. [45] discussed the $\mathrm{AI}$ in the field of healthcare. AI is continuously changing the medical practice.

This paper discusses the new concepts of the complex bipolar picture fuzzy sets (CBPFSs). Further, using the Cartesian product of CBPFS, the innovative study of complex bipolar picture fuzzy relation (CBPFR) is examined. Moreover, numerous types of CBPFRs are defined, such as reflexive, symmetric, complete, transitive, equivalence relations and equivalence classes. Each definition of CBPFRs with suitable examples and results also have been presented. The purpose of this study is to discuss some unique strategies for increasing the industries qualities by using the artificial intelligence factors. In addition, an application is provided that helps in the investigation of relationship among various industries to the key factors of artificial intelligence. They have a wide range of applications because complex valued structure can handle the multivariable difficulties. The CBPFR is the extended form of all predefined structures of bipolar fuzzy algebra such as BFR, CBFR, BIFR, CBIFR, and BPFR. The CBPFR is superior to all preexisting fuzzy structures because these structures discuss all of the three stages with both amplitude and phase term. The innovative idea of CBPFR defined all of the three stages with positive and negative effect. The membership, neutral, and nonmembership degree of the CBPFR to show both positive and negative effects of all stages with complex numbers. The concept of CBPFR is used in the other fuzzy structures, i.e., complex bipolar spherical fuzzy relation (CBSFR) and complex bipolar $\mathrm{T}$ spherical fuzzy relation (CBTSFR). Moreover, this structure can be used in all other fuzzy models such as economics, computer science, and engineering field. 
The arrangements of this paper are as follows: Section 1 presents the introduction. Section 2 describes some predefined concepts. Section 3 discusses the novelty concepts of CBPFSs, Cartesian product of two CBPFSs, CBPFRs, and their types. Section 4 consists an application of artificial intelligence investigating the relationship between industries and factor of artificial intelligence. Section 5 contains the comparison between CBPFS and preexisting structure of fuzzy algebra. Finally, Section 6 gives the conclusion.

\section{Preliminaries}

In this section, we explain some concepts of FS, CFS, IFS, CIFS, PFS, CPFS, BFS, CBFS, BIFS, and BPFS.

Definition 1 (see [1]). Let U be a universal set. Then, a fuzzy set (FS) $F_{P}$ on $U$ is expressed as

$$
F_{P}=\{(x, \phi(x): x \in \mathrm{U})\} \text {, }
$$

where $\phi: U \longrightarrow[0,1]$ is mapping of membership degree.

Definition 2 (see [22]). Let $\mathrm{U}$ be a universal set. Then, a complex fuzzy set (CFS) $F_{P}$ on $U$ is expressed as

$$
F_{P}=\left\{\left(x, \breve{\mathrm{a}}_{\phi}(x) e^{2 \pi \omega_{\phi}(x) i}\right): x \in \mathrm{U}\right\},
$$

where $\breve{a}_{\phi}, \omega_{\phi}: U \longrightarrow[0,1]$ are mappings of amplitude term and phase têrm of membership degree, respectively. Moreover, $i=\sqrt{-1}$.
Definition 3 (see [14]). Let $U$ be a universal set. Then, an intuitionistic fuzzy set (IFS) $\hat{F}_{P}$ on $U$ is expressed as

$$
F_{P}=\{x,(\phi(x), \Omega(x)): x \in U \text { U }\}
$$

where $\phi, \Omega: U \longrightarrow[0,1]$ are mappings of membership and nonmembership degrees, respectively, on condition that $0 \leq \phi(x)+\Omega(x) \leq 1$.

Definition 4 (see [26]). Let $U$ be a universal set. Then, a complex intuitionistic fuzzy set (CIFS) $F_{P}$ on $U$ is expressed as

$$
F_{P}=\left\{x,\left(\breve{\mathrm{a}}_{\phi}(x) e^{2 \pi \mathrm{\omega}_{\phi}(x) i}, \breve{\mathrm{a}}_{\Omega}(x) e^{2 \pi \mathrm{\omega}_{\Omega}(x) i}\right): x \in \mathrm{U}\right\},
$$

where $\breve{\mathrm{a}}_{\phi}, \breve{\mathrm{a}}_{\Omega}, \varpi_{\phi}, \varpi_{\Omega}: \mathrm{U} \longrightarrow[0,1]$ are mappings of amplitude terms and phase terms of membership and nonmembership degrees, respectively, on condition that $0 \leq \breve{a}_{\phi}+\breve{a}_{\Omega} \leq 1,0 \leq \varpi_{\phi}+\varpi_{\Omega} \leq 1$.

Definition 5 (see [19]). Let $U$ be a universal set. Then, a picture fuzzy set (PFS) $F_{P}$ on $\mathrm{U}$ is expressed as

$$
F_{P}=\{x,(\phi(x), \dot{\varepsilon}(x), \Omega(x)): x \in \mathrm{U}\},
$$

where $\phi, \dot{\varepsilon}, \Omega: U \longrightarrow[0,1]$ are mappings of membership, abstinence, and nonmembership degrees, respectively, on condition that $0 \leq \phi(x)+\dot{\varepsilon}(x)+\Omega(x) \leq 1$.

Definition 6 (see [29]). Let $\mathrm{U}$ be a universal set. Then, a complex picture fuzzy set (CPFS) $F_{P}$ on $U$ is expressed as

$$
F_{P}=\left\{x,\left(\breve{\mathrm{a}}_{\phi}(x) \mathrm{e}^{2 \pi \Phi_{\phi}(x) i}, \breve{\mathrm{a}}_{\dot{\varepsilon}}(\mathrm{x}) \mathrm{e}^{2 \pi \varpi_{\dot{\varepsilon}}(\mathrm{x}) \mathrm{i}}, \breve{\mathrm{a}}_{\Omega}(\mathrm{x}) \mathrm{e}^{2 \pi \Phi_{\Omega}(\mathrm{x}) \mathrm{i}}\right): x \in \mathrm{U}\right\},
$$

where $\breve{a}_{\phi}, \oplus_{\phi}, \breve{a}_{\dot{\varepsilon}}, \varpi_{\dot{\varepsilon}}, \breve{a}_{\Omega}, \oplus_{\Omega}: U \longrightarrow[0,1]$ are mappings of amplitude terms and phase terms of membership, abstinence, and nonmembership degrees, respectively, on condition that $0 \leq \breve{a}_{\phi}+\breve{a}_{\dot{\varepsilon}}+\breve{a}_{\Omega} \leq 1$ and $0 \leq{\omega_{\phi}}+{\omega_{\dot{\varepsilon}}}+\varpi_{\Omega} \leq 1$.

Definition 7 (see [31]). Let $U$ be a universal set. Then, a bipolar fuzzy set (BFS) $F_{P}$ on $U$ is expressed as

$$
F_{P}=\left\{\left(x, \phi^{+}(x), \phi^{-}(x)\right): x \in \mathrm{U}\right\},
$$

where $\phi^{+}: U \longrightarrow[0,1]$ are positive mappings of membership degree and $\phi^{-}: U \longrightarrow[-1,0]$ are negative mappings of membership degree.

Definition 8 (see [34]). Let $U$ be a universal set. Then, a

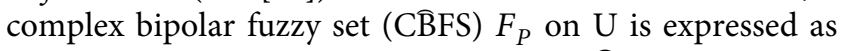

$$
F_{P}=\left\{\left(x, \breve{a}_{\phi}^{+}(x) e^{2 \pi i \omega_{\phi}^{+}(x)}, \breve{a}_{\phi}^{-}(x) e^{2 \pi i \omega_{\phi}^{-}(x)}\right): x \in \underline{U}\right\},
$$

where $\breve{a}_{\phi}^{+}, \varpi_{\phi}^{+}: U \longrightarrow[0,1]$ are positive mappings of amplitude term and phase term of membership degree, respectively, and $\breve{a}_{\phi}^{-}, \Phi_{\phi}^{-}: U \longrightarrow[-1,0]$ are negative mappings of amplitude term and phase term of membership degree, respectively.

Definition 9 (see [35]). Let $U$ be a universal set. Then, a bipolar intuitionistic fuzzy set (BIFS) $F_{P}$ on $U$ is expressed as

$$
F_{P}=\left\{x,\left(\phi^{+}(x), \phi^{-}(x)\right),\left(\Omega^{+}(x), \Omega^{-}(x)\right): x \in \mathrm{U}_{-}\right\},
$$

where $\phi^{+}, \Omega^{+}: U \longrightarrow[0,1]$ are positive mappings of membership and nonmembership degrees, respectively, and $\phi^{-}, \Omega^{-}: U \longrightarrow[-1,0]$ are negative mappings of membership and nonmembership degree, respectively, on condition that $0 \leq \phi^{+}(x)+\Omega^{+}(x) \leq 1$ and $-1 \leq \phi^{-}(x)+\Omega^{-}(x) \leq 0$.

Definition 10 (see [36]). Let $U$ be a universal set. Then, a bipolar picture fuzzy set (BPFS) $F_{P}$ on $\mathrm{U}$ is expressed as

$$
F_{P}=\left\{x,\left(\phi^{+}(x), \phi^{-}(x)\right),\left(\dot{\varepsilon}^{+}(x), \dot{\varepsilon}^{-}(x)\right),\left(\Omega^{+}(x), \Omega^{-}(x)\right): x \in \underset{-}{\mathrm{U}}\right\},
$$


where $\phi^{+}, \dot{\varepsilon}^{+}, \Omega^{+}: U \longrightarrow[0,1]$ are positive mappings of membership, abstinênce, and nonmembership degrees, respectively, and $\phi^{-}, \dot{\varepsilon}^{-}, \Omega^{-}: U \longrightarrow[-1,0]$ are negative mappings of membership, abstinence, and nonmembership degrees, respectively, on condition that $0 \leq \phi^{+}(x)+\dot{\varepsilon}^{+}(x)+$ $\Omega^{+}(x) \leq 1$ and $-1 \leq \phi^{-}(x)+\dot{\varepsilon}^{-}(x)+\Omega^{-}(x) \leq 0$.

\section{Main Results}

In this section, we define the complex bipolar intuitionistic fuzzy set (CBIFS), complex bipolar picture fuzzy set
(CBPFS), Cartesian product of two CBPFS, complex bipolar picture fuzzy relation (CBPFR), and their types.

Definition 11. Let $\mathrm{U}$ be a universal set. Then, a complex bipolar intuitionistic fuzzy set (CBIFS) $F_{P}$ on $U$ is expressed as

$$
F_{P}=\left\{x,\left(\breve{\mathrm{a}}_{\phi}^{+}(x) e^{2 \pi i \omega_{\phi}^{+}(x)}, \breve{\mathrm{a}}_{\phi}^{-}(x) e^{2 \pi i \Phi_{\phi}^{-}(x)}\right),\left(\breve{\mathrm{a}}_{\Omega}^{+}(x) e^{2 \pi i \omega_{\Omega}^{+}(x)}, \breve{\mathrm{a}}_{\Omega}^{-}(x) e^{2 \pi i \omega_{\Omega}^{-}(x)}\right): x \in \underset{-}{\mathrm{U}}\right\},
$$

where $\breve{a}_{\phi}^{+}, \varpi_{\phi}^{+}, \breve{a}_{\Omega}^{+}, \varpi_{\Omega}^{+}: U \longrightarrow[0,1]$ are positive mappings of amplitude terms and phase terms of membership and nonmembership degrees, respectively, and $\breve{a}_{\phi}^{-}, \varpi_{\phi}^{-}, \breve{a}_{\Omega}^{-}$, $\varpi_{\Omega}^{-}: \mathrm{U} \longrightarrow[-1,0]$ are negative mappings of amplitude terms and phase terms of membership and nonmembership degrees, respectively, on condition that $0 \leq \breve{a}_{\phi}^{+}+\breve{a}_{\Omega}^{+} \leq 10$ $\leq \varpi_{\phi}^{+}+\varpi_{\Omega}^{+} \leq 1,-1 \leq \breve{a}_{\phi}^{-}+\breve{a}_{\Omega}^{-} \leq 0$ and $-1 \leq \varpi_{\phi}^{-}+\varpi_{\Omega}^{-} \leq 0$.

Definition 12. Let $\mathrm{U}$ be a universal set. Then, a complex bipolar picture fuzzy set (CBPFS) $F_{P}$ on $\mathrm{U}$ is expressed as

$$
F_{P}=\left\{x,\left(\begin{array}{c}
\breve{\mathrm{a}}_{\phi}^{+}(x) e^{2 \pi i \omega_{\phi}^{+}(x)}, \\
\breve{\mathrm{a}}_{\phi}^{-}(x) e^{2 \pi i \omega_{\phi}^{-}(x)}
\end{array}\right),\left(\begin{array}{c}
\breve{\mathrm{a}}_{\dot{\varepsilon}}^{+}(x) e^{2 \pi i \omega_{\dot{\varepsilon}}^{+}(x)}, \\
\breve{\mathrm{a}}_{\dot{\varepsilon}}^{-}(x) e^{2 \pi i \omega_{\tilde{\varepsilon}}^{-}(x)}
\end{array}\right),\left(\begin{array}{c}
\breve{\mathrm{a}}_{\Omega}^{+}(x) e^{2 \pi i \omega_{\Omega}^{+}(x)}, \\
\breve{\mathrm{a}}_{\Omega}^{-}(x) e^{2 \pi i \omega_{\Omega}^{-}(x)}
\end{array}\right): x \in \underline{\mathrm{U}}\right\},
$$

where $\breve{a}_{\phi}^{+}, \varpi_{\phi}^{+}, \breve{a}_{\varepsilon}^{+}, \varpi_{\dot{\varepsilon}}^{+}, \breve{a}_{\Omega}^{+}, \varpi_{\Omega}^{+}: U \longrightarrow[0,1]$ are positive mappings of amplitude terms and phase terms of membership, abstinence, and nonmembership degrees, respectively, and $\breve{a}_{\phi}^{-}, \varpi_{\phi}^{-}, \breve{a}_{\dot{\varepsilon}}^{-}, \oplus_{\breve{a} \dot{\varepsilon}}^{-}, \breve{a}_{\Omega}^{-}, \oplus_{\Omega}^{-}: U \longrightarrow[-1,0]$ are negative mappings of amplitude terms and phase terms of membership, abstinence, and non-membership degrees, respectively, on condition that $0 \leq \breve{a}_{\phi}^{+}+\breve{a}_{\dot{\varepsilon}}^{+}+\breve{a}_{\Omega}^{+} \leq 1$,

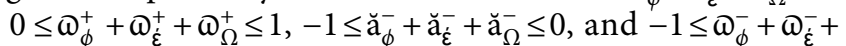
$\omega_{\Omega}^{-} \leq 0$.

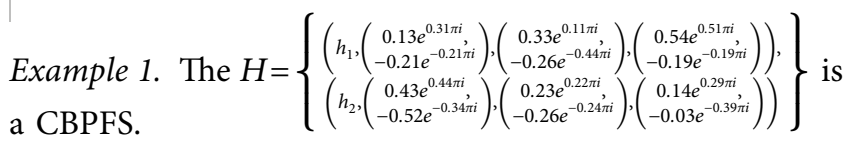

Definition 13. Take two CBPFSs

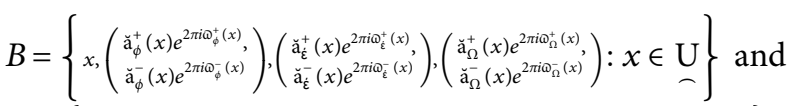

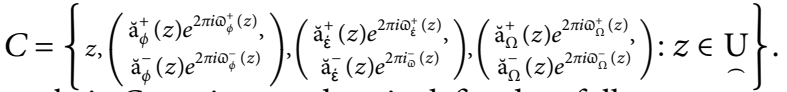

Then, their Cartesian product is defined as follows:

$$
B \times C=\left\{(x, z),\left(\begin{array}{c}
\breve{\mathrm{a}}_{\phi}^{+}(x, z) e^{2 \pi i \omega_{\phi}^{+}(x, z)}, \\
\breve{\mathrm{a}}_{\phi}^{-}(x, z) e^{2 \pi i \omega_{\phi}^{-}(x, z)}
\end{array}\right),\left(\begin{array}{c}
\breve{\mathrm{a}}_{\dot{\varepsilon}}^{+}(x, z) e^{2 \pi i \omega_{\dot{\varepsilon}}^{+}(x, z)}, \\
\breve{\mathrm{a}}_{\dot{\varepsilon}}^{-}(x, z) e^{2 \pi i \Phi_{\tilde{\varepsilon}}^{-}(x, z)}
\end{array}\right),\left(\begin{array}{c}
\breve{\mathrm{a}}_{\Omega}^{+}(x, z) e^{2 \pi i \omega_{\Omega}^{+}(x, z)}, \\
\breve{\mathrm{a}}_{\Omega}^{-}(x, z) e^{2 \pi i \omega_{\Omega}^{-}(x, z)}
\end{array}\right): x, z \in \mathrm{U}\right\},
$$

where $\quad \breve{\mathrm{a}}_{\phi}^{+}(x, z)=\min \left\{\breve{\mathrm{a}}_{\phi}^{+}(x), \breve{\mathrm{a}}_{\phi}^{+}(z)\right\} \quad \varpi_{\phi}^{+}(x, z)=\min \left\{{\varpi_{\phi}^{+}}^{+}\right.$ $\left.(x), \varpi_{\phi}^{+}(z)\right\} \quad \breve{\mathrm{a}}_{\phi}^{-}(x, z)=\max \left\{\breve{\mathrm{a}}_{\phi}^{-}(x), \breve{\mathrm{a}}_{\phi}^{-}(z)\right\} \Phi_{\phi}^{-}(x, z)=\max$ $\left\{\varpi_{\phi}^{-}(x), \Phi_{\phi}^{-}(z)\right\}, \breve{a}_{\dot{\varepsilon}}^{+}(x, z)=\min \left\{\breve{a}_{\dot{\varepsilon}}^{+}(x), \breve{a}_{\dot{\varepsilon}}^{+}(z)\right\}, \quad \Phi_{\dot{\varepsilon}}^{+}(x, z)=$ $\min \left\{\varpi_{\dot{\varepsilon}}^{+}(x), \Phi_{\dot{\varepsilon}}^{+}(z)\right\} \breve{\mathrm{a}}_{\dot{\varepsilon}}^{-}(x, z)=\max \left\{\breve{\mathrm{a}}_{\dot{\varepsilon}}^{-}(x), \breve{\mathrm{a}}_{\dot{\varepsilon}}^{-}(z)\right\},{\Phi_{\dot{\varepsilon}}^{-}}^{-}(x, z)$ $=\max \left\{\varpi_{\dot{\varepsilon}}^{-}(x), \Phi_{\dot{\varepsilon}}^{-}(z)\right\}, \breve{\mathrm{a}}_{\Omega}^{+}(x, z)=\max \left\{\breve{\mathrm{a}}_{\Omega}^{+}(x), \breve{\mathrm{a}}_{\Omega}^{+}(z)\right\} \quad \omega_{\Omega}^{+}$ $(x, z)=\max \left\{\varpi_{\Omega}^{+}(x), \varpi_{\Omega}^{+}(z)\right\} \breve{a}_{\Omega}^{-}(x, z)=\min \left\{\breve{a}_{\Omega}^{-}(x), \breve{\mathrm{a}}_{\Omega}^{-}(z)\right\}$ $\varpi_{\Omega}^{-}(x, z)=\min \left\{\Phi_{\Omega}^{-}(x), \Phi_{\Omega}^{-}(z)\right\}$.
Hence, we take ordered paired elements in the Cartesian product; the degrees of membership and abstinence are taken to be the minimum between the two pairs and the maximum nonmembership is selected for the product.

Example 2. Let a CBPFS $L$ on $F_{P}$ be defined as 


$$
L=\left\{\begin{array}{l}
\left(l_{1},\left(\begin{array}{c}
0.49 e^{0.21 \pi i}, \\
-0.11 e^{-0.35 \pi i}
\end{array}\right),\left(\begin{array}{c}
0.18 e^{0.49 \pi i}, \\
-0.39 e^{-0.37 \pi i}
\end{array}\right),\left(\begin{array}{c}
0.28 e^{0.23 \pi i}, \\
-0.19 e^{-0.27 \pi i}
\end{array}\right)\right), \\
\left(l_{2},\left(\begin{array}{c}
0.47 e^{0.33 \pi i}, \\
-0.52 e^{-0.35 \pi i}
\end{array}\right),\left(\begin{array}{c}
0.32 e^{0.25 \pi i}, \\
-0.26 e^{-0.26 \pi i}
\end{array}\right),\left(\begin{array}{c}
0.13 e^{0.37 \pi i}, \\
-0.04 e^{-0.13 \pi i}
\end{array}\right)\right), \\
\left(l_{3},\left(\begin{array}{c}
0.42 e^{0.31 \pi i}, \\
-0.23 e^{-0.04 \pi i}
\end{array}\right),\left(\begin{array}{c}
0 e^{0.28 \pi i}, \\
-0.14 e^{-0.23 \pi i}
\end{array}\right),\left(\begin{array}{c}
0.57 e^{0.03 \pi i}, \\
-0.34 e^{-0.02 \pi i}
\end{array}\right)\right), \\
\left(l_{4},\left(\begin{array}{c}
0.35 e^{0.49 \pi i}, \\
-0.37 e^{0 \pi i}
\end{array}\right),\left(\begin{array}{c}
0.43 e^{0.46 \pi i}, \\
-0.29 e^{-0.64 \pi i}
\end{array}\right),\left(\begin{array}{c}
0.17 e^{0.03 \pi i}, \\
-0.22 e^{-0.02 \pi i}
\end{array}\right)\right)
\end{array}\right\} .
$$

Then, its self-Cartesian product is

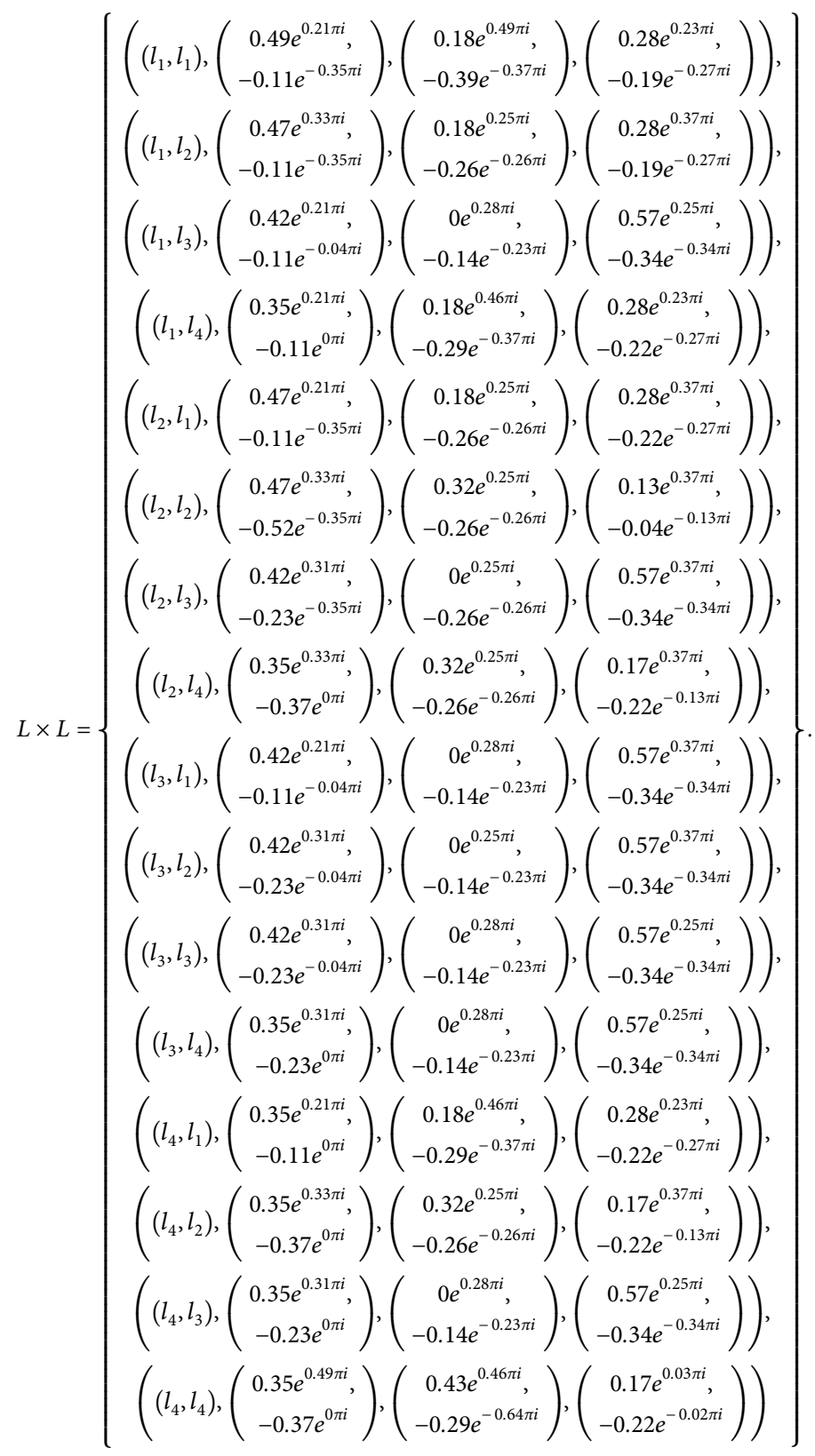


Definition 14. A complex bipolar picture fuzzy relation (CBPFR) is a subset of the Cartesian product of two complex bipolar picture fuzzy sets (CBPFSs) and is denoted by R.
Example 3. From the Cartesian product of CBPFS in equation (1), the complex bipolar picture fuzzy relation $\mathrm{R}$ is

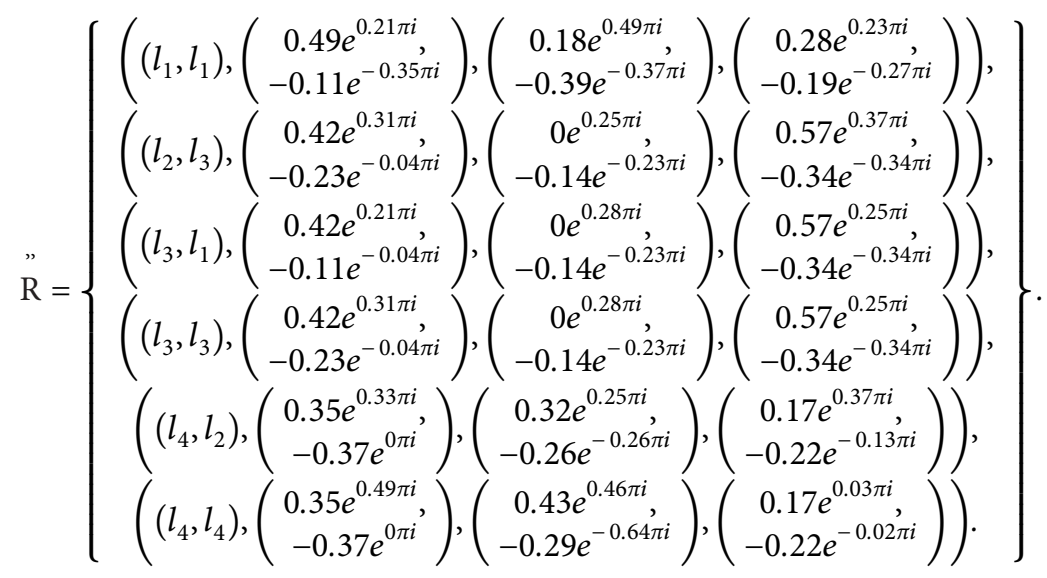

Definition 15. A CBPFR $R$ is said to be complex bipolar picture reflexive fuzzy relation (CBP reflexive FR) on a CBPFS $F_{P}$, if

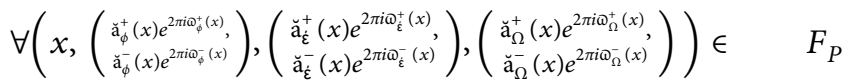

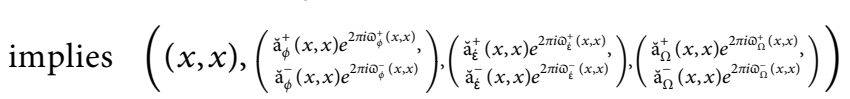
$\in$ R.
Definition 16. A CBPFR R $\mathrm{R}$ is said to be complex bipolar picture irreflexive fuzzy relation (CBP irreflexive $F R$ ) on a CBPFS $F_{P}$, if

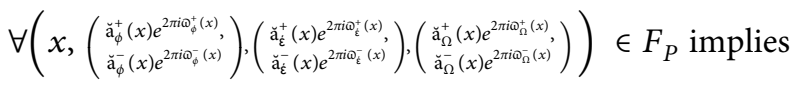

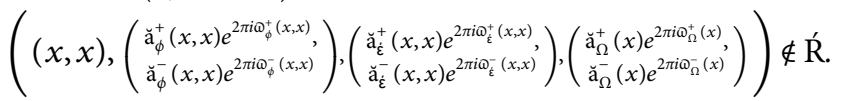

Definition 17. A CBPFR R is said to be complex bipolar picture symmetric fuzzy relation (CBP symmetric $\mathrm{FR}$ ) on a CBPFS $F_{P}$, if

$$
\begin{aligned}
& \forall\left(x,\left(\begin{array}{c}
\breve{\mathrm{a}}_{\phi}^{+}(x) e^{2 \pi i \Phi_{\phi}^{+}(x)}, \\
\breve{\mathrm{a}}_{\phi}^{-}(x) e^{2 \pi i \Phi_{\phi}^{-}(x)}
\end{array}\right),\left(\begin{array}{c}
\breve{\mathrm{a}}_{\dot{\varepsilon}}^{+}(x) e^{2 \pi i \omega_{\dot{\varepsilon}}^{+}(x)}, \\
\breve{\mathrm{a}}_{\dot{\varepsilon}}^{-}(x) e^{2 \pi i \omega_{\dot{\varepsilon}}^{-}(x)}
\end{array}\right),\left(\begin{array}{c}
\breve{\mathrm{a}}_{\Omega}^{+}(x) e^{2 \pi i \Phi_{\Omega}^{+}(x)}, \\
\breve{\mathrm{a}}_{\Omega}^{-}(x) e^{2 \pi i \omega_{\Omega}^{-}(x)}
\end{array}\right)\right),
\end{aligned}
$$

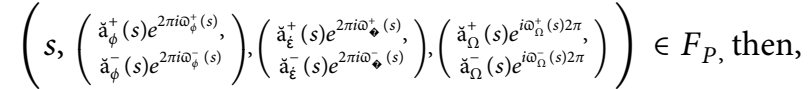

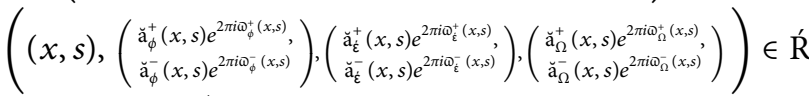

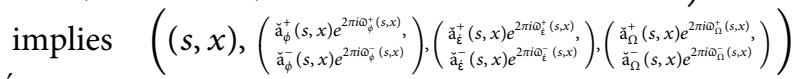

$$
\begin{aligned}
& \in \text { Ŕ. } \\
& \forall\left(x,\left(\begin{array}{c}
\breve{\mathrm{a}}_{\phi}^{+}(x) e^{2 \pi i \omega_{\phi}^{+}(x)}, \\
\breve{\mathrm{a}}_{\phi}^{-}(x) e^{2 \pi i \omega_{\phi}^{-}(x)}
\end{array}\right),\left(\begin{array}{c}
\breve{\mathrm{a}}_{\dot{\varepsilon}}^{+}(x) e^{2 \pi i \omega_{\dot{\varepsilon}}^{+}(x)}, \\
\breve{\mathrm{a}}_{\dot{\varepsilon}}^{-}(x) e^{2 \pi i \omega_{\dot{\varepsilon}}^{-}(x)}
\end{array}\right),\left(\begin{array}{c}
\breve{\mathrm{a}}_{\Omega}^{+}(x) e^{2 \pi i \omega_{\Omega}^{+}(x)}, \\
\breve{\mathrm{a}}_{\Omega}^{-}(x) e^{2 \pi i \omega_{\Omega}^{-}(x)}
\end{array}\right)\right), \\
& \left(s,\left(\begin{array}{c}
\breve{\mathrm{a}}_{\phi}^{+}(s) e^{2 \pi i \omega_{\phi}^{+}(s)}, \\
\breve{\mathrm{a}}_{\phi}^{-}(s) e^{2 \pi i \omega_{\phi}^{-}(s)}
\end{array}\right),\left(\begin{array}{c}
\breve{\mathrm{a}}_{\dot{\varepsilon}}^{+}(s) e^{2 \pi i \omega_{\dot{\varepsilon}}^{+}(s)}, \\
\breve{\mathrm{a}}_{\dot{\varepsilon}}^{-}(s) e^{2 \pi i \omega_{\dot{\varepsilon}}^{-}(s)}
\end{array}\right),\left(\begin{array}{c}
\breve{\mathrm{a}}_{\Omega}^{+}(s) e^{2 \pi i \omega_{\Omega}^{+}(s)}, \\
\breve{\mathrm{a}}_{\Omega}^{-}(s) e^{2 \pi i \omega_{\Omega}^{-}(s)}
\end{array}\right)\right),
\end{aligned}
$$

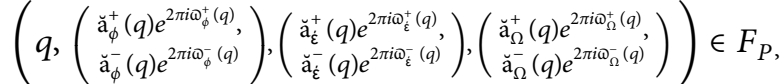

Definition 18. A CBPFR R $\mathrm{R}$ is said to be complex bipolar picture transitive fuzzy relation (CBP transitive $\mathrm{FR}$ ) on a

then 


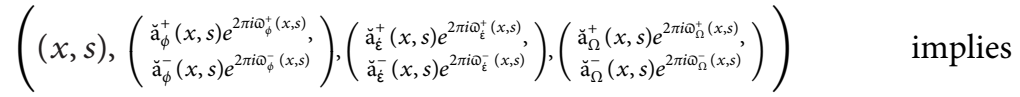

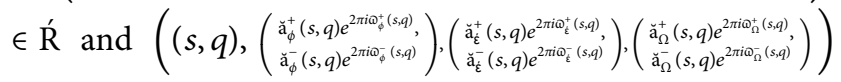

$$
\begin{aligned}
& \in \dot{R} \\
& \left((x, q),\left(\begin{array}{c}
\breve{a}_{\phi}^{+}(x, q) e^{2 \pi i \omega_{\phi}^{+}(x, q)}, \\
\breve{a}_{\phi}^{-}(x, q) e^{2 \pi i \Phi_{\phi}^{-}(x, q)}
\end{array}\right),\left(\begin{array}{c}
\breve{a}_{\dot{\varepsilon}}^{+}(x, q) e^{2 \pi i \omega_{\dot{\varepsilon}}^{+}(x, q)}, \\
\breve{a}_{\dot{\varepsilon}}^{-}(x, q) e^{2 \pi i \omega_{\dot{\varepsilon}}^{-}(x, q)}
\end{array}\right),\left(\begin{array}{c}
\breve{a}_{\Omega}^{+}(x, q) e^{2 \pi i \omega_{\Omega}^{+}(x, q)}, \\
\breve{a}_{\Omega}^{-}(x, q) e^{2 \pi i \omega_{\Omega}^{-}(x, q)}
\end{array}\right)\right) \in \dot{\mathrm{R}} .
\end{aligned}
$$

Definition 19. A CBPFR R is said to be complex bipolar picture equivalence fuzzy relation (CBP equivalence $\mathrm{FR}$ ) on a CBPFS $F_{P}$, if it is

(i) $\mathrm{CBP}$ reflexive $\mathrm{FR}$

(ii) CBP symmetric FR (iii) $\mathrm{CBP}$ transitive FR

Example 4. From the Cartesian product of CBPFS in equation (1), the complex bipolar picture equivalence fuzzy relation $\mathrm{R}$ is,

$$
\mathrm{R}=\left\{\begin{array}{l}
\left(\left(l_{1}, l_{1}\right),\left(\begin{array}{c}
0.49 e^{0.21 \pi i}, \\
-0.11 e^{-0.35 \pi i}
\end{array}\right),\left(\begin{array}{c}
0.18 e^{0.49 \pi i}, \\
-0.39 e^{-0.37 \pi i}
\end{array}\right),\left(\begin{array}{c}
0.28 e^{0.23 \pi i}, \\
-0.19 e^{-0.27 \pi i}
\end{array}\right)\right), \\
\left(\left(l_{1}, l_{2}\right),\left(\begin{array}{c}
0.47 e^{0.21 \pi i}, \\
-0.11 e^{-0.35 \pi i}
\end{array}\right),\left(\begin{array}{c}
0.18 e^{0.25 \pi i}, \\
-0.26 e^{-0.26 \pi i}
\end{array}\right),\left(\begin{array}{c}
0.28 e^{0.37 \pi i}, \\
-0.19 e^{-0.27 \pi i}
\end{array}\right)\right), \\
\left(\left(l_{1}, l_{3}\right),\left(\begin{array}{c}
0.42 e^{0.21 \pi i}, \\
-0.11 e^{-0.04 \pi i}
\end{array}\right),\left(\begin{array}{c}
0 e^{0.28 \pi i}, \\
-0.14 e^{-0.23 \pi i}
\end{array}\right),\left(\begin{array}{c}
0.57 e^{0.25 \pi i}, \\
-0.34 e^{-0.34 \pi i}
\end{array}\right)\right), \\
\left(\left(l_{2}, l_{1}\right),\left(\begin{array}{c}
0.47 e^{0.21 \pi i}, \\
-0.11 e^{-0.35 \pi i}
\end{array}\right),\left(\begin{array}{c}
0.18 e^{0.25 \pi i}, \\
-0.26 e^{-0.26 \pi i}
\end{array}\right),\left(\begin{array}{c}
0.28 e^{0.37 \pi i}, \\
-0.19 e^{-0.27 \pi i}
\end{array}\right)\right), \\
\left(\left(l_{2}, l_{2}\right),\left(\begin{array}{c}
0.47 e^{0.33 \pi i}, \\
-0.52 e^{-0.35 \pi i}
\end{array}\right),\left(\begin{array}{c}
0.32 e^{0.25 \pi i}, \\
-0.26 e^{-0.26 \pi i}
\end{array}\right),\left(\begin{array}{c}
0.13 e^{0.37 \pi i}, \\
-0.04 e^{-0.13 \pi i}
\end{array}\right)\right), \\
\left(\left(l_{2}, l_{3}\right),\left(\begin{array}{c}
0.42 e^{0.31 \pi i}, \\
-0.23 e^{-0.04 \pi i}
\end{array}\right),\left(\begin{array}{c}
0 e^{0.25 \pi i}, \\
-0.14 e^{-0.23 \pi i}
\end{array}\right),\left(\begin{array}{c}
0.57 e^{0.37 \pi i}, \\
-0.34 e^{-0.34 \pi i}
\end{array}\right)\right), \\
\left(\left(l_{3}, l_{1}\right),\left(\begin{array}{c}
0.42 e^{0.21 \pi i}, \\
-0.11 e^{-0.04 \pi i}
\end{array}\right),\left(\begin{array}{c}
0 e^{0.28 \pi i}, \\
-0.14 e^{-0.23 \pi i}
\end{array}\right),\left(\begin{array}{c}
0.57 e^{0.25 \pi i}, \\
-0.34 e^{-0.34 \pi i}
\end{array}\right)\right), \\
\left(\left(l_{3}, l_{2}\right),\left(\begin{array}{c}
0.42 e^{0.31 \pi i}, \\
-0.23 e^{-0.04 \pi i}
\end{array}\right),\left(\begin{array}{c}
0 e^{0.25 \pi i}, \\
-0.14 e^{-0.23 \pi i}
\end{array}\right),\left(\begin{array}{c}
0.57 e^{0.37 \pi i}, \\
-0.34 e^{-0.34 \pi i}
\end{array}\right)\right), \\
\left(\left(l_{3}, l_{3}\right),\left(\begin{array}{c}
0.42 e^{0.31 \pi i}, \\
-0.23 e^{-0.04 \pi i}
\end{array}\right),\left(\begin{array}{c}
0 e^{0.28 \pi i}, \\
-0.14 e^{-0.23 \pi i}
\end{array}\right),\left(\begin{array}{c}
0.57 e^{0.25 \pi i}, \\
-0.34 e^{-0.34 \pi i}
\end{array}\right)\right), \\
\left(\left(l_{4}, l_{4}\right),\left(\begin{array}{c}
0.35 e^{0.49 \pi i}, \\
-0.37 e^{0 \pi i}
\end{array}\right),\left(\begin{array}{c}
0.43 e^{0.46 \pi i}, \\
-0.29 e^{-0.64 \pi i}
\end{array}\right),\left(\begin{array}{c}
0.17 e^{0.03 \pi i}, \\
-0.22 e^{-0.02 \pi i}
\end{array}\right)\right) .
\end{array}\right\} .
$$

Definition 20. A CBPFR $R$ is said to be complex bipolar picture preorder fuzzy relation (CBP preorder FR) on a CBPFS $F_{P}$, if it is

(i) $\mathrm{CBP}$ reflexive FR

\section{(ii) CBP transitive FR}

Example 5. From the Cartesian product of CBPFS in equation (1), the complex bipolar picture preorder fuzzy relation $R$ is, 


$$
\mathrm{R}=\left\{\begin{array}{l}
\left(\left(l_{1}, l_{1}\right),\left(\begin{array}{c}
0.49 e^{0.21 \pi i}, \\
-0.11 e^{-0.35 \pi i}
\end{array}\right),\left(\begin{array}{c}
0.18 e^{0.49 \pi i}, \\
-0.39 e^{-0.37 \pi i}
\end{array}\right),\left(\begin{array}{c}
0.28 e^{0.23 \pi i}, \\
-0.19 e^{-0.27 \pi i}
\end{array}\right)\right), \\
\left(\left(l_{1}, l_{2}\right),\left(\begin{array}{c}
0.47 e^{0.21 \pi i}, \\
-0.11 e^{-0.35 \pi i}
\end{array}\right),\left(\begin{array}{c}
0.18 e^{0.25 \pi i}, \\
-0.26 e^{-0.26 \pi i}
\end{array}\right),\left(\begin{array}{c}
0.28 e^{0.37 \pi i}, \\
-0.19 e^{-0.27 \pi i}
\end{array}\right)\right), \\
\left(\left(l_{1}, l_{3}\right),\left(\begin{array}{c}
0.42 e^{0.21 \pi i}, \\
-0.11 e^{-0.04 \pi i}
\end{array}\right),\left(\begin{array}{c}
0 e^{0.28 \pi i}, \\
-0.14 e^{-0.23 \pi i}
\end{array}\right),\left(\begin{array}{c}
0.57 e^{0.25 \pi i}, \\
-0.34 e^{-0.34 \pi i}
\end{array}\right)\right), \\
\left(\left(l_{2}, l_{2}\right),\left(\begin{array}{c}
0.47 e^{0.33 \pi i}, \\
-0.52 e^{-0.35 \pi i}
\end{array}\right),\left(\begin{array}{c}
0.32 e^{0.25 \pi i}, \\
-0.26 e^{-0.26 \pi i}
\end{array}\right),\left(\begin{array}{c}
0.13 e^{0.37 \pi i}, \\
-0.04 e^{-0.13 \pi i}
\end{array}\right)\right), \\
\left(\left(l_{2}, l_{3}\right),\left(\begin{array}{c}
0.42 e^{0.31 \pi i}, \\
-0.23 e^{-0.04 \pi i}
\end{array}\right),\left(\begin{array}{c}
0 e^{0.25 \pi i}, \\
-0.14 e^{-0.23 \pi i}
\end{array}\right),\left(\begin{array}{c}
0.57 e^{0.37 \pi i}, \\
-0.34 e^{-0.34 \pi i}
\end{array}\right)\right), \\
\left(\left(l_{3}, l_{3}\right),\left(\begin{array}{c}
0.42 e^{0.31 \pi i}, \\
-0.23 e^{-0.04 \pi i}
\end{array}\right),\left(\begin{array}{c}
0 e^{0.28 \pi i}, \\
-0.14 e^{-0.23 \pi i}
\end{array}\right),\left(\begin{array}{c}
0.57 e^{0.25 \pi i}, \\
-0.34 e^{-0.34 \pi i}
\end{array}\right)\right), \\
\left(\left(l_{4}, l_{4}\right),\left(\begin{array}{c}
0.35 e^{0.49 \pi i}, \\
-0.37 e^{0 \pi i}
\end{array}\right),\left(\begin{array}{c}
0.43 e^{0.46 \pi i}, \\
-0.29 e^{-0.64 \pi i}
\end{array}\right),\left(\begin{array}{c}
0.17 e^{0.03 \pi i}, \\
-0.22 e^{-0.02 \pi i}
\end{array}\right)\right)
\end{array}\right\} .
$$

Definition 21. A CBPFR Ŕ is said to be complex bipolar picture antisymmetric fuzzy relation (CBP antisymmetric

FR) on a CBPFS $F_{P}$, if

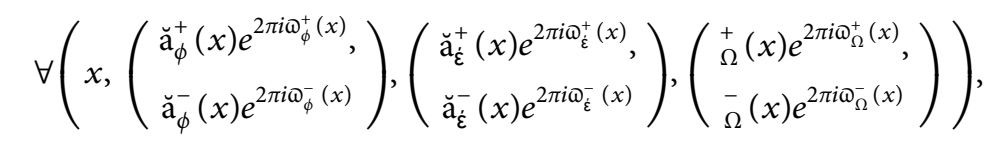

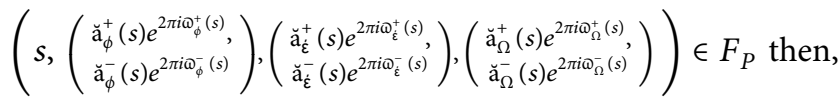

$$
\begin{aligned}
& \left((x, s),\left(\begin{array}{c}
\breve{\mathrm{a}}_{\phi}^{+}(x, s) e^{2 \pi i \omega_{\phi}^{+}(x, s)}, \\
\breve{\mathrm{a}}_{\phi}^{-}(x, s) e^{2 \pi i \omega_{\phi}^{-}(x, s)}
\end{array}\right),\left(\begin{array}{c}
\breve{\mathrm{a}}_{\dot{\varepsilon}}^{+}(x, s) e^{2 \pi i \omega_{\dot{\varepsilon}}^{+}(x, s)}, \\
\breve{\mathrm{a}}_{\dot{\varepsilon}}^{-}(x, s) e^{2 \pi i \Phi_{\bar{\varepsilon}}^{-}(x, s)}
\end{array}\right),\left(\begin{array}{c}
\breve{\mathrm{a}}_{\Omega}^{+}(x, s) e^{2 \pi i \omega_{\Omega}^{+}(x, s)}, \\
\breve{\mathrm{a}}_{\Omega}^{-}(x, s) e^{2 \pi i \omega_{\Omega}^{-}(x, s)}
\end{array}\right)\right) \in \dot{\mathrm{R}}, \\
& \left((s, x),\left(\begin{array}{c}
\breve{\mathrm{a}}_{\phi}^{+}(s, x) e^{2 \pi i \omega_{\phi}^{+}(s, x)}, \\
\breve{\mathrm{a}}_{\phi}^{-}(s, x) e^{2 \pi i \omega_{\phi}^{-}(s, x)}
\end{array}\right),\left(\begin{array}{c}
\breve{\mathrm{a}}_{\dot{\varepsilon}}^{+}(s, x) e^{2 \pi i \omega_{\dot{\varepsilon}}^{+}(s, x)}, \\
\breve{\mathrm{a}}_{\dot{\varepsilon}}^{-}(s, x) e^{2 \pi i \omega_{\dot{\varepsilon}}^{-}(s, x)}
\end{array}\right),\left(\begin{array}{c}
\breve{\mathrm{a}}_{\Omega}^{+}(s, x) e^{2 \pi i \omega_{\Omega}^{+}(s, x)}, \\
\breve{\mathrm{a}}_{\Omega}^{-}(s, x) e^{2 \pi i \omega_{\Omega}^{-}(s, x)}
\end{array}\right)\right) \in \text { Ŕ implies, } \\
& \left((x, s),\left(\begin{array}{c}
\breve{\mathrm{a}}_{\phi}^{+}(x, s) e^{2 \pi i \omega_{\phi}^{+}(x, s)}, \\
\breve{\mathrm{a}}_{\phi}^{-}(x, s) e^{2 \pi i \omega_{\phi}^{-}(x, s)}
\end{array}\right),\left(\begin{array}{c}
\breve{\mathrm{a}}_{\dot{\varepsilon}}^{+}(x, s) e^{2 \pi i \omega_{\dot{\varepsilon}}^{+}(x, s)}, \\
\breve{\mathrm{a}}_{\dot{\varepsilon}}^{-}(x, s) e^{2 \pi i \omega_{\dot{\varepsilon}}^{-}(x, s)}
\end{array}\right),\left(\begin{array}{c}
\breve{\mathrm{a}}_{\Omega}^{+}(x, s) e^{2 \pi i \omega_{\Omega}^{+}(x, s)}, \\
\breve{\mathrm{a}}_{\Omega}^{-}(x, s) e^{2 \pi i \omega_{\Omega}^{-}(x, s)}
\end{array}\right)\right), \\
& =\left((s, x),\left(\begin{array}{c}
\breve{\mathrm{a}}_{\phi}^{+}(s, x) e^{2 \pi i \omega_{\phi}^{+}(s, x)}, \\
\breve{\mathrm{a}}_{\phi}^{-}(s, x) e^{2 \pi i \omega_{\phi}^{-}(s, x)}
\end{array}\right),\left(\begin{array}{c}
\breve{\mathrm{a}}_{\dot{\varepsilon}}^{+}(s, x) e^{2 \pi i \omega_{\tilde{\varepsilon}}^{+}(s, x)}, \\
\breve{\mathrm{a}}_{\dot{\varepsilon}}^{-}(s, x) e^{2 \pi i \omega_{\dot{\varepsilon}}^{-}(s, x)}
\end{array}\right),\left(\begin{array}{c}
\breve{\mathrm{a}}_{\Omega}^{+}(s, x) e^{2 \pi i \omega_{\Omega}^{+}(s, x)}, \\
\breve{\mathrm{a}}_{\Omega}^{-}(s, x) e^{2 \pi i \omega_{\Omega}^{-}(s, x)}
\end{array}\right)\right) .
\end{aligned}
$$

Definition 22. A CBPFR Ŕ is said to be complex bipolar picture partial order fuzzy relation (CBP partial order FR) on a CBPFS $F_{P}$, if it is

(i) $\mathrm{CBP}$ reflexive $\mathrm{FR}$

(ii) CBP antisymmetric FR (iii) CBP transitive FR

Example 6. From the Cartesian product of CBPFS in equation (1), the complex bipolar picture partial order fuzzy relation $R^{\prime}$ is, 


$$
\mathrm{R}=\left\{\begin{array}{l}
\left(\left(l_{1}, l_{1}\right),\left(\begin{array}{c}
0.49 e^{0.21 \pi i}, \\
-0.11 e^{-0.35 \pi i}
\end{array}\right),\left(\begin{array}{c}
0.18 e^{0.49 \pi i}, \\
-0.39 e^{-0.37 \pi i}
\end{array}\right),\left(\begin{array}{c}
0.28 e^{0.23 \pi i}, \\
-0.19 e^{-0.27 \pi i}
\end{array}\right)\right), \\
\left(\left(l_{1}, l_{3}\right),\left(\begin{array}{c}
0.42 e^{0.21 \pi i}, \\
-0.11 e^{-0.04 \pi i}
\end{array}\right),\left(\begin{array}{c}
0 e^{0.28 \pi i}, \\
-0.14 e^{-0.23 \pi i}
\end{array}\right),\left(\begin{array}{c}
0.57 e^{0.25 \pi i}, \\
-0.34 e^{-0.34 \pi i}
\end{array}\right)\right), \\
\left(\left(l_{1}, l_{4}\right),\left(\begin{array}{c}
0.35 e^{0.21 \pi i}, \\
-0.11 e^{0 \pi i}
\end{array}\right),\left(\begin{array}{c}
0.18 e^{0.46 \pi i}, \\
-0.29 e^{-0.37 \pi i}
\end{array}\right),\left(\begin{array}{c}
0.28 e^{0.23 \pi i}, \\
-0.22 e^{-0.27 \pi i}
\end{array}\right)\right), \\
\left(\left(l_{2}, l_{2}\right),\left(\begin{array}{c}
0.47 e^{0.33 \pi i}, \\
-0.52 e^{-0.35 \pi i}
\end{array}\right),\left(\begin{array}{c}
0.32 e^{0.25 \pi i}, \\
-0.26 e^{-0.26 \pi i}
\end{array}\right),\left(\begin{array}{c}
0.13 e^{0.37 \pi i}, \\
-0.04 e^{-0.13 \pi i}
\end{array}\right)\right), \\
\left(\left(l_{3}, l_{4}\right),\left(\begin{array}{c}
0.35 e^{0.31 \pi i}, \\
-0.23 e^{0 \pi i}
\end{array}\right),\left(\begin{array}{c}
0 e^{0.28 \pi i}, \\
-0.14 e^{-0.23 \pi i}
\end{array}\right),\left(\begin{array}{c}
0.57 e^{0.25 \pi i}, \\
-0.34 e^{-0.34 \pi i}
\end{array}\right)\right), \\
\left(\left(l_{3}, l_{3}\right),\left(\begin{array}{c}
0.42 e^{0.31 \pi i}, \\
-0.23 e^{-0.04 \pi i}
\end{array}\right),\left(\begin{array}{c}
0 e^{0.28 \pi i}, \\
-0.14 e^{-0.23 \pi i}
\end{array}\right),\left(\begin{array}{c}
0.57 e^{0.25 \pi i}, \\
-0.34 e^{-0.34 \pi i}
\end{array}\right)\right), \\
\left(\left(l_{4}, l_{4}\right),\left(\begin{array}{c}
0.35 e^{0.49 \pi i}, \\
-0.37 e^{0 \pi i}
\end{array}\right),\left(\begin{array}{c}
0.43 e^{0.46 \pi i}, \\
-0.29 e^{-0.64 \pi i}
\end{array}\right),\left(\begin{array}{c}
0.17 e^{0.03 \pi i}, \\
-0.22 e^{-0.02 \pi i}
\end{array}\right)\right) .
\end{array}\right\} .
$$

Definition 23. A CBPFR R is said to be complex bipolar picture complete fuzzy relation (CBP complete FR) on a CBPFS $F_{P}$, if

$$
\begin{aligned}
& \forall\left(x,\left(\begin{array}{c}
\breve{\mathrm{a}}_{\phi}^{+}(x) e^{2 \pi i \varpi_{\phi}^{+}(x)}, \\
\breve{\mathrm{a}}_{\phi}^{-}(x) e^{2 \pi i \Phi_{\phi}^{-}(x)}
\end{array}\right),\left(\begin{array}{c}
\breve{\mathrm{a}}_{\dot{\varepsilon}}^{+}(x) e^{2 \pi i \varpi_{\dot{\varepsilon}}^{+}(x)}, \\
\breve{\mathrm{a}}_{\dot{\varepsilon}}^{-}(x) e^{2 \pi i \varpi_{\dot{\varepsilon}}^{-}(x)}
\end{array}\right),\left(\begin{array}{c}
\breve{\mathrm{a}}_{\Omega}^{+}(x) e^{2 \pi i \omega_{\Omega}^{+}(x)}, \\
\breve{\mathrm{a}}_{\Omega}^{-}(x) e^{2 \pi i \Phi_{\Omega}^{-}(x)}
\end{array}\right)\right), \\
& \left(s,\left(\begin{array}{c}
\breve{\mathrm{a}}_{\phi}^{+}(s) e^{2 \pi i \omega_{\phi}^{+}(s)}, \\
\breve{\mathrm{a}}_{\phi}^{-}(s) e^{2 \pi i \Phi_{\phi}^{-}(s)}
\end{array}\right),\left(\begin{array}{c}
\breve{\mathrm{a}}_{\dot{\varepsilon}}^{+}(s) e^{2 \pi i \omega_{\dot{\varepsilon}}^{+}(s)}, \\
\breve{\mathrm{a}}_{\dot{\varepsilon}}^{-}(s) e^{2 \pi i \Phi_{\dot{\varepsilon}}^{-}(s)}
\end{array}\right),\left(\begin{array}{c}
\breve{\mathrm{a}}_{\Omega}^{+}(s) e^{2 \pi i \Phi_{\Omega}^{+}(s)}, \\
\breve{\mathrm{a}}_{\Omega}^{-}(s) e^{2 \pi i \Phi_{\Omega}^{-}(s)}
\end{array}\right)\right) \in F_{P}, \\
& \operatorname{Either}\left((x, s),\left(\begin{array}{c}
\breve{\mathrm{a}}_{\phi}^{+}(x, s) e^{2 \pi i \omega_{\phi}^{+}(x, s)}, \\
\breve{\mathrm{a}}_{\phi}^{-}(x, s) e^{2 \pi i \varpi_{\phi}^{-}(x, s)}
\end{array}\right),\left(\begin{array}{c}
\breve{\mathrm{a}}_{\dot{\varepsilon}}^{+}(x, s) e^{2 \pi i \varpi_{\dot{\varepsilon}}^{+}(x, s)}, \\
\breve{\mathrm{a}}_{\dot{\varepsilon}}^{-}(x, s) e^{2 \pi i \varpi_{\dot{\varepsilon}}^{-}(x, s)}
\end{array}\right),\left(\begin{array}{c}
\breve{\mathrm{a}}_{\Omega}^{+}(x, s) e^{2 \pi i \varpi_{\Omega}^{+}(x, s)}, \\
\breve{\mathrm{a}}_{\Omega}^{-}(x, s) e^{2 \pi i \varpi_{\Omega}^{-}(x, s)}
\end{array}\right)\right) \in \mathrm{R}^{\prime} \\
& \operatorname{Or}\left((s, x),\left(\begin{array}{c}
\breve{\mathrm{a}}_{\phi}^{+}(s, x) e^{2 \pi i \Phi_{\phi}^{+}(s, x)}, \\
\breve{\mathrm{a}}_{\phi}^{-}(s, x) e^{2 \pi i \Phi_{\phi}^{-}(s, x)}
\end{array}\right),\left(\begin{array}{c}
\breve{\mathrm{a}}_{\dot{\varepsilon}}^{+}(s, x) e^{2 \pi i \omega_{\dot{\varepsilon}}^{+}(s, x)}, \\
\breve{\mathrm{a}}_{\dot{\varepsilon}}^{-}(s, x) e^{2 \pi i \Phi_{\dot{\varepsilon}}^{-}(s, x)}
\end{array}\right),\left(\begin{array}{c}
\breve{\mathrm{a}}_{\Omega}^{+}(s, x) e^{2 \pi i \Phi_{\Omega}^{+}(s, x)}, \\
\breve{\mathrm{a}}_{\Omega}^{-}(s, x) e^{2 \pi i \Phi_{\Omega}^{-}(s, x)}
\end{array}\right)\right) \in \hat{\mathrm{R}} .
\end{aligned}
$$

Definition 24. A CBPFR Ŕ is said to be complex bipolar picture strict order fuzzy relation (CBP strict order FR) on a CBPFS $F_{P}$, if it is

(i) CBP irreflexive FR

\section{(ii) CBP transitive FR}

Example 7. From the Cartesian product of CBPFS in equation (1), the complex bipolar picture strict order fuzzy relation $\mathrm{R}$ is, 


$$
, \quad \mathrm{R}=\left\{\begin{aligned}
\left(\left(l_{1}, l_{2}\right),\left(\begin{array}{c}
0.47 e^{0.21 \pi i}, \\
-0.11 e^{-0.35 \pi i}
\end{array}\right),\left(\begin{array}{c}
0.18 e^{0.25 \pi i}, \\
-0.26 e^{-0.26 \pi i}
\end{array}\right),\left(\begin{array}{c}
0.28 e^{0.37 \pi i}, \\
-0.19 e^{-0.27 \pi i}
\end{array}\right)\right), \\
\left(\left(l_{1}, l_{4}\right),\left(\begin{array}{c}
0.35 e^{0.21 \pi i}, \\
-0.11 e^{0 \pi i}
\end{array}\right),\left(\begin{array}{c}
0.18 e^{0.46 \pi i}, \\
-0.29 e^{-0.37 \pi i}
\end{array}\right),\left(\begin{array}{c}
0.28 e^{0.23 \pi i}, \\
-0.22 e^{-0.27 \pi i}
\end{array}\right)\right), \\
\left(\left(l_{4}, l_{2}\right),\left(\begin{array}{c}
0.35 e^{0.33 \pi i}, \\
-0.37 e^{0 \pi i}
\end{array}\right),\left(\begin{array}{c}
0.32 e^{0.25 \pi i}, \\
-0.26 e^{-0.26 \pi i}
\end{array}\right),\left(\begin{array}{c}
0.17 e^{0.37 \pi i}, \\
-0.22 e^{-0.13 \pi i}
\end{array}\right)\right) .
\end{aligned}\right\}
$$

Definition 25. A CBPFR R $\mathrm{R}$ is said to be complex bipolar picture linear order fuzzy relation (CBP linear order FR) on a CBPFS $F_{P}$, if it is
(i) $\mathrm{CBP}$ reflexive $\mathrm{FR}$
(ii) CBP antisymmetric FR
(iii) CBP transitive FR
(iv) CBP complete FR

Definition 26. A CBPFR $\mathrm{R}$ is said to be complex bipolar picture inverse fuzzy relation (CBP inverse FR) on a CBPFS $F_{P}$, if

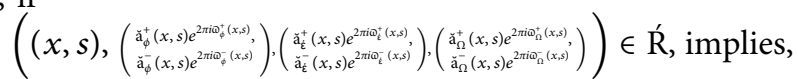

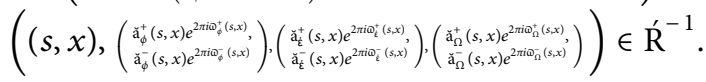

Definition 27. Let $\mathrm{R}_{1}$ and $\mathrm{R}_{2}$ be two CBPF relation on a CBPFS $F_{P}$. Then, the complex bipolar picture composite FR $\mathrm{R}_{1} \circ \mathrm{R}_{2}$ is defined as

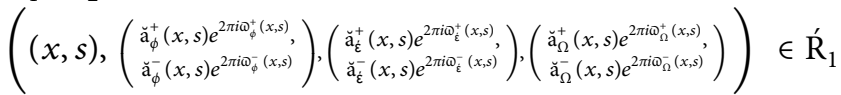

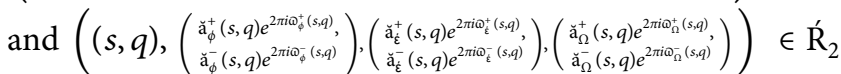

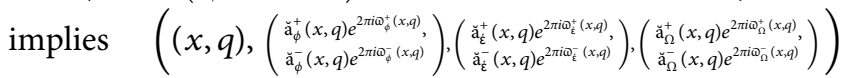
$\in \mathrm{R}_{1} \circ \mathrm{R}_{2}$.

Definition 28. A CBPF equivalence class of $x \bmod \mathrm{R}$ is expressed as

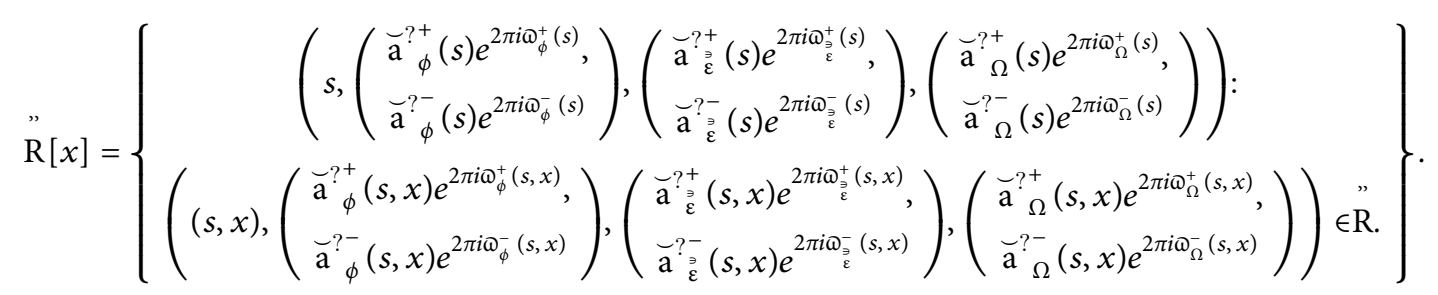

Example 8. From the Cartesian product of CBPFS in equation (1), consider the complex bipolar picture equivalence fuzzy relation $\mathrm{R}$, 


$$
\Rightarrow=\left\{\begin{array}{l}
\left(\left(l_{1}, l_{1}\right),\left(\begin{array}{c}
0.49 e^{0.21 \pi i}, \\
-0.11 e^{-0.35 \pi i}
\end{array}\right),\left(\begin{array}{c}
0.18 e^{0.49 \pi i}, \\
-0.39 e^{-0.37 \pi i}
\end{array}\right),\left(\begin{array}{c}
0.28 e^{0.23 \pi i}, \\
-0.19 e^{-0.27 \pi i}
\end{array}\right)\right), \\
\left(\left(l_{2}, l_{2}\right),\left(\begin{array}{c}
0.47 e^{0.33 \pi i}, \\
-0.52 e^{-0.35 \pi i}
\end{array}\right),\left(\begin{array}{c}
0.32 e^{0.25 \pi i}, \\
-0.26 e^{-0.26 \pi i}
\end{array}\right),\left(\begin{array}{c}
0.13 e^{0.37 \pi i}, \\
-0.04 e^{-0.13 \pi i}
\end{array}\right)\right), \\
\left(\left(l_{2}, l_{3}\right),\left(\begin{array}{c}
0.42 e^{0.31 \pi i}, \\
-0.23 e^{-0.04 \pi i}
\end{array}\right),\left(\begin{array}{c}
0 e^{0.25 \pi i}, \\
-0.14 e^{-0.23 \pi i}
\end{array}\right),\left(\begin{array}{c}
0.57 e^{0.37 \pi i}, \\
-0.34 e^{-0.34 \pi i}
\end{array}\right)\right), \\
\left(\left(l_{3}, l_{2}\right),\left(\begin{array}{c}
0.42 e^{0.31 \pi i}, \\
-0.23 e^{-0.04 \pi i}
\end{array}\right),\left(\begin{array}{c}
0 e^{0.25 \pi i}, \\
-0.14 e^{-0.23 \pi i}
\end{array}\right),\left(\begin{array}{c}
0.57 e^{0.37 \pi i}, \\
-0.34 e^{-0.34 \pi i}
\end{array}\right)\right), \\
\left(\left(l_{3}, l_{3}\right),\left(\begin{array}{c}
0.42 e^{0.31 \pi i}, \\
-0.23 e^{-0.04 \pi i}
\end{array}\right),\left(\begin{array}{c}
0 e^{0.28 \pi i}, \\
-0.14 e^{-0.23 \pi i}
\end{array}\right),\left(\begin{array}{c}
0.57 e^{0.25 \pi i}, \\
-0.34 e^{-0.34 \pi i}
\end{array}\right)\right), \\
\left(\left(l_{4}, l_{4}\right),\left(\begin{array}{c}
0.35 e^{0.49 \pi i}, \\
-0.37 e^{0 \pi i}
\end{array}\right),\left(\begin{array}{c}
0.43 e^{0.46 \pi i}, \\
-0.29 e^{-0.64 \pi i}
\end{array}\right),\left(\begin{array}{c}
0.17 e^{0.03 \pi i}, \\
-0.22 e^{-0.02 \pi i}
\end{array}\right)\right) .
\end{array}\right\} .
$$

Now, complex bipolar fuzzy equivalence classes are:

$$
\begin{aligned}
& \hat{\mathrm{R}}\left[\mathrm{l}_{1}\right]=\left\{l_{1},\left(\begin{array}{c}
0.49 e^{0.21 \pi i}, \\
-0.11 e^{-0.35 \pi i}
\end{array}\right),\left(\begin{array}{c}
0.18 e^{0.49 \pi i}, \\
-0.39 e^{-0.37 \pi i}
\end{array}\right),\left(\begin{array}{c}
0.28 e^{0.23 \pi i}, \\
-0.19 e^{-0.27 \pi i}
\end{array}\right)\right\}, \\
& \dot{\mathrm{R}}\left[\mathrm{l}_{2}\right]=\left\{\begin{array}{l}
\left(l_{2},\left(\begin{array}{c}
0.47 e^{0.33 \pi i}, \\
-0.52 e^{-0.35 \pi i}
\end{array}\right),\left(\begin{array}{c}
0.32 e^{0.25 \pi i}, \\
-0.26 e^{-0.26 \pi i}
\end{array}\right),\left(\begin{array}{c}
0.13 e^{0.37 \pi i}, \\
-0.04 e^{-0.13 \pi i}
\end{array}\right)\right), \\
\left(l_{3},\left(\begin{array}{c}
0.42 e^{0.31 \pi i}, \\
-0.23 e^{-0.04 \pi i}
\end{array}\right),\left(\begin{array}{c}
0 e^{0.28 \pi i}, \\
-0.14 e^{-0.23 \pi i}
\end{array}\right),\left(\begin{array}{c}
0.57 e^{0.25 \pi i}, \\
-0.34 e^{-0.34 \pi i}
\end{array}\right)\right),
\end{array}\right\} \\
& \dot{\mathrm{R}}\left[\mathrm{l}_{3}\right]=\left\{\begin{array}{l}
\left(l_{2},\left(\begin{array}{c}
0.47 e^{0.33 \pi i}, \\
-0.52 e^{-0.35 \pi i}
\end{array}\right),\left(\begin{array}{c}
0.32 e^{0.25 \pi i}, \\
-0.26 e^{-0.26 \pi i}
\end{array}\right),\left(\begin{array}{c}
0.13 e^{0.37 \pi i}, \\
-0.04 e^{-0.13 \pi i}
\end{array}\right)\right), \\
\left(l_{3},\left(\begin{array}{c}
0.42 e^{0.31 \pi i}, \\
-0.23 e^{-0.04 \pi i}
\end{array}\right),\left(\begin{array}{c}
0 e^{0.28 \pi i}, \\
-0.14 e^{-0.23 \pi i}
\end{array}\right),\left(\begin{array}{c}
0.57 e^{0.25 \pi i}, \\
-0.34 e^{-0.34 \pi i}
\end{array}\right)\right),
\end{array}\right\} \\
& \hat{\mathrm{R}}\left[\mathrm{l}_{4}\right]=\left\{\left(l_{4},\left(\begin{array}{c}
0.35 e^{0.49 \pi i}, \\
-0.37 e^{0 \pi i}
\end{array}\right),\left(\begin{array}{c}
0.43 e^{0.46 \pi i}, \\
-0.29 e^{-0.64 \pi i}
\end{array}\right),\left(\begin{array}{c}
0.17 e^{0.03 \pi i}, \\
-0.22 e^{-0.02 \pi i}
\end{array}\right)\right)\right\} .
\end{aligned}
$$

Theorem 1. A CBPFR Ŕ on $F_{p}$ is a CBP symmetric FR ŔR $=$ $\hat{R}^{-1}$.
Proof. Necessity condition Assume that $\dot{R}^{\prime}=\dot{\mathrm{R}}^{-1}$. Then,

$$
\begin{aligned}
& \left((x, s),\left(\begin{array}{c}
\breve{\mathrm{a}}_{\phi}^{+}(x, s) e^{2 \pi i \omega_{\phi}^{+}(x, s)}, \\
\breve{\mathrm{a}}_{\phi}^{-}(x, s) e^{2 \pi i \omega_{\phi}^{-}(x, s)}
\end{array}\right),\left(\begin{array}{c}
\breve{\mathrm{a}}_{\dot{\varepsilon}}^{+}(x, s) e^{2 \pi i \omega_{\dot{\varepsilon}}^{+}(x, s)}, \\
\breve{\mathrm{a}}_{\dot{\varepsilon}}^{-}(x, s) e^{2 \pi i \omega_{\tilde{\varepsilon}}^{-}(x, s)}
\end{array}\right),\left(\begin{array}{c}
\breve{\mathrm{a}}_{\Omega}^{+}(x, s) e^{2 \pi i \omega_{\Omega}^{+}(x, s)}, \\
\breve{\mathrm{a}}_{\Omega}^{-}(x, s) e^{2 \pi i \omega_{\Omega}^{-}(x, s)}
\end{array}\right)\right) \in \dot{\mathrm{R}} \\
& \Leftrightarrow\left((s, x),\left(\begin{array}{c}
\breve{\mathrm{a}}_{\phi}^{+}(s, x) e^{2 \pi i \omega_{\phi}^{+}(s, x)}, \\
\breve{\mathrm{a}}_{\phi}^{-}(s, x) e^{2 \pi i \omega_{\phi}^{-}(s, x)}
\end{array}\right),\left(\begin{array}{c}
\breve{\mathrm{a}}_{\dot{\varepsilon}}^{+}(s, x) e^{2 \pi i \omega_{\dot{\varepsilon}}^{+}(s, x)}, \\
\breve{\mathrm{a}}_{\dot{\varepsilon}}^{-}(s, x) e^{2 \pi i \omega_{\tilde{\varepsilon}}^{-}(s, x)}
\end{array}\right),\left(\begin{array}{c}
\breve{\mathrm{a}}_{\Omega}^{+}(s, x) e^{2 \pi i \omega_{\Omega}^{+}(s, x)}, \\
\breve{\mathrm{a}}_{\Omega}^{-}(s, x) e^{2 \pi i \omega_{\Omega}^{-}(s, x)}
\end{array}\right)\right) \in \dot{\mathrm{R}}^{-1} .
\end{aligned}
$$

Since $R^{\prime}=\dot{R}^{-1}$. Thus, 


$$
\Leftrightarrow\left((s, x),\left(\begin{array}{c}
\breve{\mathrm{a}}_{\phi}^{+}(s, x) e^{2 \pi i \Phi_{\phi}^{+}(s, x)}, \\
\breve{\mathrm{a}}_{\phi}^{-}(s, x) e^{2 \pi i \Phi_{\phi}^{-}(s, x)}
\end{array}\right),\left(\begin{array}{c}
\breve{\mathrm{a}}_{\dot{\varepsilon}}^{+}(s, x) e^{2 \pi i \omega_{\dot{\varepsilon}}^{+}(s, x)}, \\
\breve{\mathrm{a}}_{\dot{\varepsilon}}^{-}(s, x) e^{2 \pi i \omega_{\dot{\varepsilon}}^{-}(s, x)}
\end{array}\right),\left(\begin{array}{c}
\breve{\mathrm{a}}_{\Omega}^{+}(s, x) e^{2 \pi i \omega_{\Omega}^{+}(s, x)}, \\
\breve{\mathrm{a}}_{\Omega}^{-}(s, x) e^{2 \pi i \Phi_{\Omega}^{-}(s, x)}
\end{array}\right)\right) \in \hat{\mathrm{R}} .
$$

Therefore, by the statement of theorem, $R$ is a complex bipolar picture symmetric fuzzy relation on $F_{p}$.

Sufficient Condition
Assume that $\mathrm{R}$ is a complex bipolar picture symmetric fuzzy relation on $F_{p}$, then

$$
\begin{aligned}
& \left((x, s),\left(\begin{array}{c}
\breve{\mathrm{a}}_{\phi}^{+}(x, s) e^{2 \pi i \omega_{\phi}^{+}(x, s)}, \\
\breve{\mathrm{a}}_{\phi}^{-}(x, s) e^{2 \pi i \omega_{\phi}^{-}(x, s)}
\end{array}\right),\left(\begin{array}{c}
\breve{\mathrm{a}}_{\dot{\varepsilon}}^{+}(x, s) e^{2 \pi i \omega_{\dot{\varepsilon}}^{+}(x, s)}, \\
\breve{\mathrm{a}}_{\dot{\varepsilon}}^{-}(x, s) e^{2 \pi i \omega_{\dot{\varepsilon}}^{-}(x, s)}
\end{array}\right),\left(\begin{array}{c}
\breve{\mathrm{a}}_{\Omega}^{+}(x, s) e^{2 \pi i \omega_{\Omega}^{+}(x, s)}, \\
\breve{\mathrm{a}}_{\Omega}^{-}(x, s) e^{2 \pi i \omega_{\Omega}^{-}(x, s)}
\end{array}\right)\right) \in \mathrm{R}, \\
& \Leftrightarrow\left((s, x),\left(\begin{array}{c}
\breve{\mathrm{a}}_{\phi}^{+}(s, x) e^{2 \pi i \omega_{\phi}^{+}(s, x)}, \\
\breve{\mathrm{a}}_{\phi}^{-}(s, x) e^{2 \pi i \omega_{\phi}^{-}(s, x)}
\end{array}\right),\left(\begin{array}{c}
\breve{\mathrm{a}}_{\dot{\varepsilon}}^{+}(s, x) e^{2 \pi i \omega_{\dot{\varepsilon}}^{+}(s, x)} \\
\breve{\mathrm{a}}_{\dot{\varepsilon}}^{-}(s, x) e^{2 \pi i \omega_{\dot{\varepsilon}}^{-}(s, x)}
\end{array}\right),\left(\begin{array}{c}
\breve{\mathrm{a}}_{\Omega}^{+}(s, x) e^{2 \pi i \omega_{\Omega}^{+}(s, x)}, \\
\breve{\mathrm{a}}_{\Omega}^{-}(s, x) e^{2 \pi i \omega_{\Omega}^{-}(s, x)}
\end{array}\right)\right) \in \mathrm{R}^{\prime} .
\end{aligned}
$$

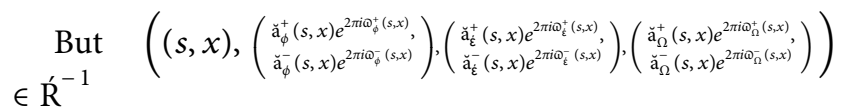

Thus, $\mathrm{R}^{\prime}=\mathrm{R}^{-1}$. Hence, theorem is proved.

Theorem 2. A CBPFR $R$ on $F_{p}$ is a CBP transitive $F R$ $\dot{R} \circ R \subseteq R$.

Proof. Necessity condition

Assume that $\mathrm{R}$ is a complex bipolar picture transitive fuzzy relation on a CBPFS $F_{p}$.

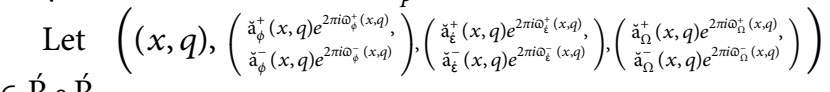

$\in \mathrm{R} \circ \mathrm{R}$

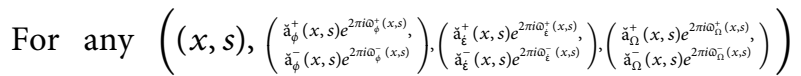

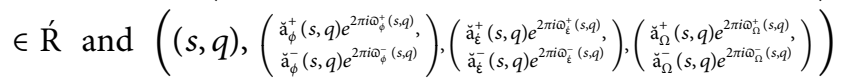

$\in \mathrm{R}^{\prime}$ $\in$ R.

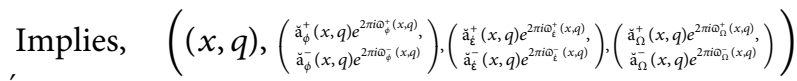

Since RoR R $\subseteq$ R.

Sufficient condition:

Conversely suppose that ŔR $\subseteq R$, then

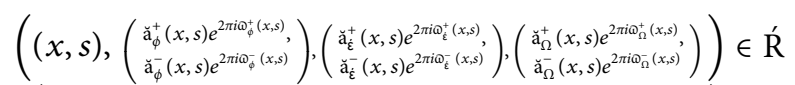

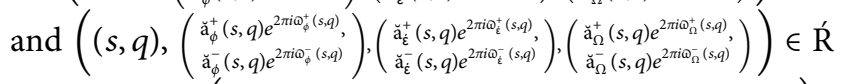
$\mathrm{R} \circ \mathrm{R}$

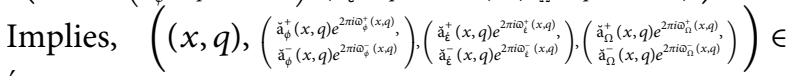

But it is assumed that $\mathrm{R} \circ \mathrm{R} \subseteq \mathrm{R}$. thus,

$$
\left((x, q),\left(\begin{array}{c}
\breve{\mathrm{a}}_{\phi}^{+}(x, q) e^{2 \pi i \omega_{\phi}^{+}(x, q)}, \\
\breve{\mathrm{a}}_{\phi}^{-}(x, q) e^{2 \pi i \omega_{\phi}^{-}(x, q)}
\end{array}\right),\left(\begin{array}{c}
\breve{\mathrm{a}}_{\dot{\varepsilon}}^{+}(x, q) e^{2 \pi i \omega_{\dot{\varepsilon}}^{+}(x, q)}, \\
\breve{\mathrm{a}}_{\dot{\varepsilon}}^{-}(x, q) e^{2 \pi i \Phi_{\dot{\varepsilon}}^{-}(x, q)}
\end{array}\right),\left(\begin{array}{c}
\breve{\mathrm{a}}_{\Omega}^{+}(x, q) e^{2 \pi i \Phi_{\Omega}^{+}(x, q)}, \\
\breve{\mathrm{a}}_{\Omega}^{-}(x, q) e^{2 \pi i \omega_{\Omega}^{-}(x, q)}
\end{array}\right)\right) \in \dot{\mathrm{R}} .
$$

Hence, $\mathrm{R}^{\prime}$ is a complex bipolar picture transitive fuzzy relation on a CBPFS $F_{p}$.

Theorem 3. A CBPFR $R$ on $F_{p}$ is a CBP equivalence $F R$ $R ́ R \circ R=R$.

Proof. Necessity condition

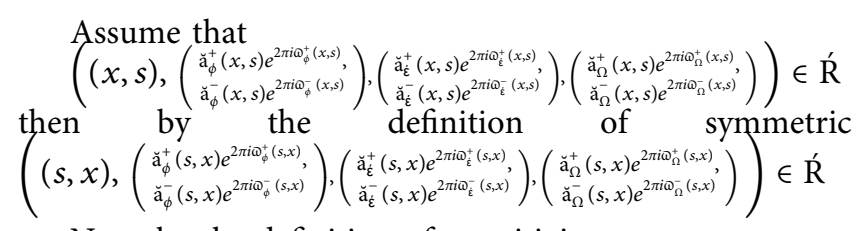

Now, by the definition of transitivity,

$$
\left((x, x),\left(\begin{array}{c}
\breve{\mathrm{a}}_{\phi}^{+}(x, x) e^{2 \pi i \omega_{\phi}^{+}(x, x)}, \\
\breve{\mathrm{a}}_{\phi}^{-}(x, x) e^{2 \pi i \omega_{\phi}^{-}(x, x)}
\end{array}\right),\left(\begin{array}{c}
\breve{\mathrm{a}}_{\dot{\varepsilon}}^{+}(x, x) e^{2 \pi i \omega_{\dot{\varepsilon}}^{+}(x, x)}, \\
\breve{\mathrm{a}}_{\dot{\varepsilon}}^{-}(x, x) e^{2 \pi i \omega_{\dot{\varepsilon}}^{-}(x, x)}
\end{array}\right),\left(\begin{array}{c}
\breve{\mathrm{a}}_{\Omega}^{+}(x) e^{2 \pi i \omega_{\Omega}^{+}(x)}, \\
\breve{\mathrm{a}}_{\Omega}^{-}(x) e^{2 \pi i \omega_{\Omega}^{-}(x)}
\end{array}\right)\right) \in \dot{\mathrm{R}} .
$$


Also, the definition of the composition,

$$
\left((x, x),\left(\begin{array}{c}
\breve{\mathrm{a}}_{\phi}^{+}(x, x) e^{2 \pi i \varpi_{\phi}^{+}(x, x)}, \\
\breve{\mathrm{a}}_{\phi}^{-}(x, x) e^{2 \pi i \omega_{\phi}^{-}(x, x)}
\end{array}\right),\left(\begin{array}{c}
\breve{\mathrm{a}}_{\dot{\varepsilon}}^{+}(x, x) e^{2 \pi i \omega_{\dot{\varepsilon}}^{+}(x, x)}, \\
\breve{\mathrm{a}}_{\dot{\varepsilon}}^{-}(x, x) e^{2 \pi i \omega_{\dot{\varepsilon}}^{-}(x, x)}
\end{array}\right),\left(\begin{array}{c}
\breve{\mathrm{a}}_{\Omega}^{+}(x) e^{2 \pi i \omega_{\Omega}^{+}(x)}, \\
\breve{\mathrm{a}}_{\Omega}^{-}(x) e^{2 \pi i \omega_{\Omega}^{-}(x)}
\end{array}\right)\right) \in \dot{\mathrm{R}} \circ \mathrm{R},
$$

Therefore, ŔŔ

Sufficient Condition

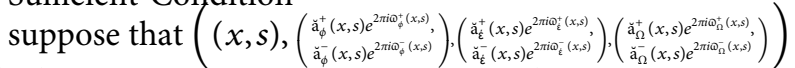
$\in \mathrm{R}^{\circ} \circ \mathrm{R}$ then there exist $q \in \mathrm{R}$ Such that,

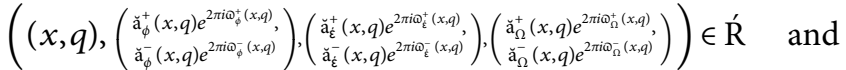

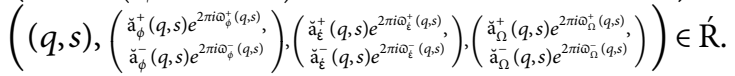

$$
\left((x, s),\left(\begin{array}{c}
\breve{\mathrm{a}}_{\phi}^{+}(x, s) e^{2 \pi i \omega_{\phi}^{+}(x, s)}, \\
\breve{\mathrm{a}}_{\phi}^{-}(x, s) e^{2 \pi i \omega_{\phi}^{-}(x, s)}
\end{array}\right),\left(\begin{array}{c}
\breve{\mathrm{a}}^{+}(x, s) e^{2 \pi i \omega_{\dot{\varepsilon}}^{+}(x, s)} \\
\breve{\mathrm{a}}^{-}(x, s) e^{2 \pi i \omega_{\dot{\varepsilon}}^{-}(x, s)}
\end{array}\right),\left(\begin{array}{c}
\breve{\mathrm{a}}_{\Omega}^{+}(x, s) e^{2 \pi i \omega_{\Omega}^{+}(x, s)}, \\
\breve{\mathrm{a}}_{\Omega}^{-}(x, s) e^{2 \pi i \omega_{\Omega}^{-}(x, s)}
\end{array}\right)\right) \in \mathrm{R}^{\prime},
$$$$
\Rightarrow \mathrm{R} \circ \mathrm{R} \subseteq a ̆ R \text {. }
$$

Hence, implies that
Thus, equations (35) and (36)

$$
\mathrm{R} \circ \mathrm{R}=\mathrm{R} \text {. }
$$

Theorem 4. The inverse of the CBP partial order fuzzy relation $R$ on $F_{p}$ is again a CBP partial order fuzzy relation $R$ on $F_{p}$.
Proof. The complex bipolar picture partial order fuzzy relation Ŕ on a CBPFS $F_{p}$; Ŕ satisfies the properties of complex bipolar picture reflexive, antisymmetric and transitive fuzzy relations.

Since, $\mathrm{R}^{\mathrm{R}}$ is a complex bipolar picture reflexive fuzzy relation. For any $x \in \mathrm{R}^{\prime}$

$$
\Rightarrow\left((x, x),\left(\begin{array}{c}
\breve{\mathrm{a}}_{\phi}^{+}(x, x) e^{2 \pi i \omega_{\phi}^{+}(x, x)}, \\
\breve{\mathrm{a}}_{\phi}^{-}(x, x) e^{2 \pi i \Phi_{\phi}^{-}(x, x)}
\end{array}\right),\left(\begin{array}{c}
\breve{\mathrm{a}}_{\dot{\varepsilon}}^{+}(x, x) e^{2 \pi i \omega_{\dot{\varepsilon}}^{+}(x, x)}, \\
\breve{\mathrm{a}}_{\dot{\varepsilon}}^{-}(x, x) e^{2 \pi i \omega_{\dot{\varepsilon}}^{-}(x, x)}
\end{array}\right),\left(\begin{array}{c}
\breve{\mathrm{a}}_{\Omega}^{+}(x) e^{2 \pi i \omega_{\Omega}^{+}(x)}, \\
\breve{\mathrm{a}}_{\Omega}^{-}(x) e^{2 \pi i \omega_{\Omega}^{-}(x)}
\end{array}\right)\right) \in \mathrm{R} .
$$

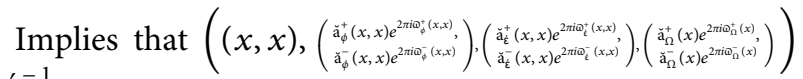
$\in \mathrm{R}^{-1}$

Hence, $\mathrm{R}^{-1}$ is also a complex bipolar picture reflexive fuzzy relation.

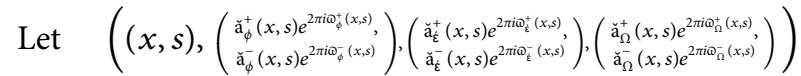

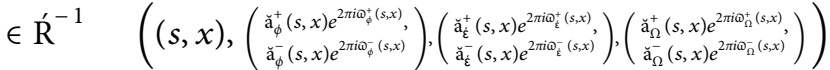

$\in \mathrm{R}^{-1}$. Then, by the definition of inverse

$$
\begin{aligned}
& \Rightarrow\left((x, s),\left(\begin{array}{c}
\breve{\mathrm{a}}_{\phi}^{+}(x, s) e^{2 \pi i \omega_{\phi}^{+}(x, s)}, \\
\breve{\mathrm{a}}_{\phi}^{-}(x, s) e^{2 \pi i \omega_{\phi}^{-}(x, s)}
\end{array}\right),\left(\begin{array}{c}
\breve{\mathrm{a}}_{\dot{\varepsilon}}^{+}(x, s) e^{2 \pi i \omega_{\tilde{\varepsilon}}^{+}(x, s)} \\
\check{\mathrm{a}}_{\dot{\varepsilon}}^{-}(x, s) e^{2 \pi i \omega_{\tilde{\varepsilon}}^{-}(x, s)}
\end{array}\right),\left(\begin{array}{c}
\breve{\mathrm{a}}_{\Omega}^{+}(x, s) e^{2 \pi i \omega_{\Omega}^{+}(x, s)} \\
\check{\mathrm{a}}_{\Omega}^{-}(x, s) e^{2 \pi i \omega_{\Omega}^{-}(x, s)},
\end{array}\right)\right) \in \mathrm{R}^{\prime}
\end{aligned}
$$

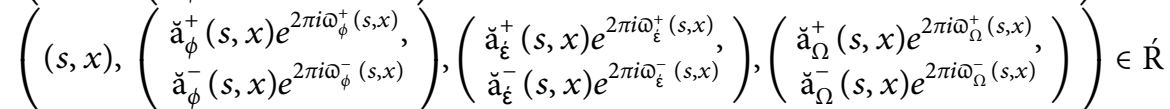

So, $\mathrm{R}$ is a complex bipolar picture antisymmetric fuzzy relation. Therefore, 


$$
\begin{aligned}
& \left((x, s),\left(\begin{array}{c}
\breve{\mathrm{a}}_{\phi}^{+}(x, s) e^{2 \pi i \omega_{\phi}^{+}(x, s)}, \\
\breve{\mathrm{a}}_{\phi}^{-}(x, s) e^{2 \pi i \omega_{\phi}^{-}(x, s)}
\end{array}\right),\left(\begin{array}{c}
\breve{\mathrm{a}}_{\dot{\varepsilon}}^{+}(x, s) e^{2 \pi i \omega_{\dot{\varepsilon}}^{+}(x, s)}, \\
\breve{\mathrm{a}}_{\dot{\varepsilon}}^{-}(x, s) e^{2 \pi i \omega_{\dot{\varepsilon}}^{-}(x, s)}
\end{array}\right),\left(\begin{array}{c}
\breve{\mathrm{a}}_{\Omega}^{+}(x, s) e^{2 \pi i \Phi_{\Omega}^{+}(x, s)}, \\
\breve{\mathrm{a}}_{\Omega}^{-}(x, s) e^{2 \pi i \omega_{\Omega}^{-}(x, s)}
\end{array}\right)\right) \\
& =\left((s, x),\left(\begin{array}{c}
\breve{\mathrm{a}}_{\phi}^{+}(s, x) e^{2 \pi i \omega_{\phi}^{+}(s, x)}, \\
\breve{\mathrm{a}}_{\phi}^{-}(s, x) e^{2 \pi i \omega_{\phi}^{-}(s, x)}
\end{array}\right),\left(\begin{array}{c}
\breve{\mathrm{a}}_{\dot{\varepsilon}}^{+}(s, x) e^{2 \pi i \omega_{\dot{\varepsilon}}^{+}(s, x)}, \\
\breve{\mathrm{a}}_{\dot{\varepsilon}}^{-}(s, x) e^{2 \pi i \omega_{\dot{\varepsilon}}^{-}(s, x)}
\end{array}\right),\left(\begin{array}{c}
\breve{\mathrm{a}}_{\Omega}^{+}(s, x) e^{2 \pi i \Phi_{\Omega}^{+}(s, x)}, \\
\breve{\mathrm{a}}_{\Omega}^{-}(s, x) e^{2 \pi i \omega_{\Omega}^{-}(s, x)}
\end{array}\right)\right) .
\end{aligned}
$$

Hence, $\mathrm{R}^{-1}$ is also a complex bipolar picture antisymmetric fuzzy relation.

Let,

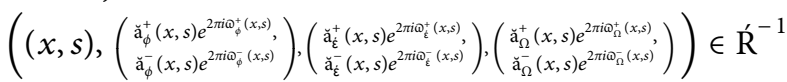

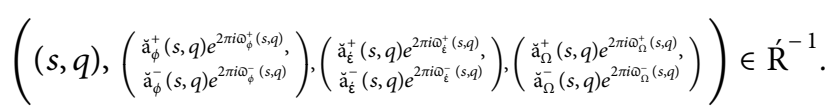

Then,

$$
\begin{aligned}
& \Rightarrow\left((q, s),\left(\begin{array}{c}
\breve{\mathrm{a}}_{\phi}^{+}(q, s) e^{2 \pi i \omega_{\phi}^{+}(q, s)}, \\
\bar{\phi}(q, s) e^{2 \pi i \omega_{\phi}^{-}(q, s)}
\end{array}\right),\left(\begin{array}{c}
\breve{\mathrm{a}}_{\dot{\varepsilon}}^{+}(q, s) e^{2 \pi i \omega_{\dot{\varepsilon}}^{+}(q, s)}, \\
\breve{\mathrm{a}}_{\dot{\varepsilon}}^{-}(q, s) e^{2 \pi i \omega_{\dot{\varepsilon}}^{-}(q, s)}
\end{array}\right),\left(\begin{array}{c}
\breve{\mathrm{a}}_{\Omega}^{+}(q, s) e^{2 \pi i \omega_{\Omega}^{+}(q, s)}, \\
\breve{\mathrm{a}}_{\Omega}^{-}(q, s) e^{2 \pi i \omega_{\Omega}^{-}(q, s)}
\end{array}\right)\right) \in \mathrm{R}^{\prime} \\
& \left((s, x),\left(\begin{array}{c}
\breve{\mathrm{a}}_{\phi}^{+}(s, x) e^{2 \pi i \omega_{\phi}^{+}(s, x)}, \\
\breve{\mathrm{a}}_{\phi}^{-}(s, x) e^{2 \pi i \omega_{\phi}^{-}(s, x)}
\end{array}\right),\left(\begin{array}{c}
\breve{\mathrm{a}}_{\dot{\varepsilon}}^{+}(s, x) e^{2 \pi i \omega_{\dot{\varepsilon}}^{+}(s, x)}, \\
\breve{\mathrm{a}}_{\dot{\varepsilon}}^{-}(s, x) e^{2 \pi i \omega_{\dot{\varepsilon}}^{-}(s, x)}
\end{array}\right),\left(\begin{array}{c}
\breve{\mathrm{a}}_{\Omega}^{+}(s, x) e^{2 \pi i \omega_{\Omega}^{+}(s, x)}, \\
\breve{\mathrm{a}}_{\Omega}^{-}(s, x) e^{2 \pi i \omega_{\Omega}^{-}(s, x)}
\end{array}\right)\right) \in \mathrm{R}^{\prime} .
\end{aligned}
$$

So, $\mathrm{R}$ is a complex bipolar picture transitive fuzzy relation. Therefore,

$$
\begin{aligned}
& \left((q, x),\left(\begin{array}{c}
\breve{\mathrm{a}}_{\phi}^{+}(q, x) e^{2 \pi i \Phi_{\phi}^{+}(q, x)}, \\
\breve{\mathrm{a}}_{\phi}^{-}(q, x) e^{2 \pi i \Phi_{\phi}^{-}(q, x)}
\end{array}\right),\left(\begin{array}{c}
\breve{\mathrm{a}}_{\dot{\varepsilon}}^{+}(q, x) e^{2 \pi i \omega_{\dot{\varepsilon}}^{+}(q, x)}, \\
\breve{\mathrm{a}}_{\dot{\varepsilon}}^{-}(q, x) e^{2 \pi i \Phi_{\dot{\varepsilon}}^{-}(q, x)}
\end{array}\right),\left(\begin{array}{c}
\breve{\mathrm{a}}_{\Omega}^{+}(q, x) e^{2 \pi i \Phi_{\Omega}^{+}(q, x)}, \\
\breve{\mathrm{a}}_{\Omega}^{-}(q, x) e^{2 \pi i \Phi_{\Omega}^{-}(q, x)}
\end{array}\right)\right) \in \mathrm{R}^{\prime} \\
& \Rightarrow\left((x, q),\left(\begin{array}{c}
\breve{\mathrm{a}}_{\phi}^{+}(x, q) e^{2 \pi i \Phi_{\phi}^{+}(x, q)}, \\
\breve{\mathrm{a}}_{\phi}^{-}(x, q) e^{2 \pi i \Phi_{\phi}^{-}(x, q)}
\end{array}\right),\left(\begin{array}{c}
\breve{\mathrm{a}}_{\dot{\varepsilon}}^{+}(x, q) e^{2 \pi i \Phi_{\dot{\varepsilon}}^{+}(x, q)}, \\
\breve{\mathrm{a}}_{\dot{\varepsilon}}^{-}(x, q) e^{2 \pi i \Phi_{\dot{\varepsilon}}^{-}(x, q)}
\end{array}\right),\left(\begin{array}{c}
\breve{\mathrm{a}}_{\Omega}^{+}(x, q) e^{2 \pi i \Phi_{\Omega}^{+}(x, q)}, \\
\breve{\mathrm{a}}_{\Omega}^{-}(x, q) e^{2 \pi i \Phi_{\Omega}^{-}(x, q)}
\end{array}\right)\right) \in \mathrm{R}^{-1} .
\end{aligned}
$$

Hence, $\mathrm{R}^{-1}$ is also a complex bipolar picture transitive fuzzy relation.

Thus, prove that $\mathrm{R}^{-1}$ is also a complex bipolar picture partial order fuzzy relation on $F_{p}$.

\section{Application}

In this section, CBPFSs and CBPFRs are used to study the artificial intelligence factors and industries.

4.1. Artificial Intelligence (AI). Artificial intelligence is the simulation of human intelligence processes by machines, especially computer systems [40-45]. The goals of AI include reasoning, knowledge representation, planning, learning, perception and the ability to move and manipulate objects. AI has assisted people living independently with disabilities. The impact of artificial intelligence on society has been largely positive, bringing contributions that have made life easier for us humans, from being able to store and analyze data in multiple industries effectively, to improve our regular routines with virtual and home assistant. AI is moving fast-paced across industries. AI is not a single technology, it is an umbrella terms that includes any types of software or hardware components that support machine learning, computer vision, neutral language processing. Figure 1 depicts the application procedures.

Figure 1 show the application procedures. Firstly, we discuss some artificial intelligence factors and examine some industries that are using the artificial intelligence factors. Hence, each factor of artificial intelligence and industries has been assigned the positive and negative membership degree, positive and negative abstinence degree, and positive and negative non-membership degree. The positive membership degree shows the likeliness of effectiveness and negative membership degree show the unlikeliness of effectiveness. The positive neutral degree shows the likeliness of neutral effect and negative neutral degree show the unlikeliness of neutral effect. The positive non-membership degree shows the likeliness of ineffectiveness and negative non-membership degree show the unlikeliness of ineffectiveness. Then define the relation between artificial intelligence factors and industries to discuss the Cartesian product. Finally, they read the information.

4.2. AI Technologies. Some key elements are discussed that need to be understood in artificial intelligence. Table 1 represents the summary of artificial intelligence technologies/factors. 


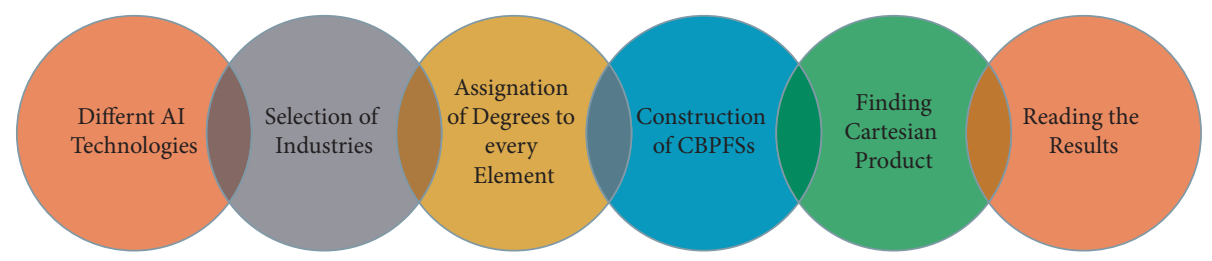

Figure 1: Application procedures.

TABLE 1: Summary of artificial intelligence factors.

\begin{tabular}{lc}
\hline Factors & Abbreviation \\
\hline Machine learning & ML \\
Neutral language processing & NLP \\
Expert system & ES \\
Computer vision & $\mathrm{CV}$ \\
Conversational AI & CAI \\
\hline
\end{tabular}

4.2.1. Machine Learning ( $M L)$. Machine learning is the basic foundation of artificial intelligence. Most AI solutions depend on machine learning algorithms. It is defined as the capability of a machine to imitate intelligent human behavior.

$$
\left(M L,\left(\begin{array}{c}
0.45 e^{0.31 \pi i}, \\
-0.23 e^{-0.21 \pi i}
\end{array}\right),\left(\begin{array}{c}
0.28 e^{0.43 \pi i}, \\
-0.14 e^{-0.34 \pi i}
\end{array}\right),\left(\begin{array}{c}
0.27 e^{0.11 \pi i}, \\
-0.59 e^{-0.19 \pi i}
\end{array}\right)\right)
$$

4.2.2. Neutral Language Processing (NLP). It can analyze, understand the language that people speak and write in everyday life. This basically, is software that understands the written and spoken languages.

$$
\left(N L P,\left(\begin{array}{c}
0.53 e^{0.41 \pi i}, \\
-0.34 e^{-0.11 \pi i}
\end{array}\right),\left(\begin{array}{c}
0.33 e^{0.21 \pi i}, \\
-0.24 e^{-0.24 \pi i}
\end{array}\right),\left(\begin{array}{c}
0.08 e^{0.21 \pi i}, \\
-0.40 e^{-0.17 \pi i}
\end{array}\right)\right) \text {. }
$$

4.2.3. Expert System (ES). Expert systems gain knowledge about a specific subject and can solve problems as accurately as a human expert on this subject. An expert system also called a knowledge-based system, is a computer program designed to stimulate the problem-solving behavior of an expert in a narrow domain.

$$
\left(E S,\left(\begin{array}{c}
0.68 e^{0.51 \pi i}, \\
-0.44 e^{-0.01 \pi i}
\end{array}\right),\left(\begin{array}{c}
0.27 e^{0.29 \pi i}, \\
-0.23 e^{-0.14 \pi i}
\end{array}\right),\left(\begin{array}{c}
0.01 e^{0.16 \pi i}, \\
-0.29 e^{-0.20 \pi i}
\end{array}\right)\right) .
$$

4.2.4. Computer Vision ( $C V)$. Computer vision is another subset of AI that deals with image processing and visual processing. It allows a machine to scan an image and use comparative analysis to identify objects in the image.

$$
\left(C V,\left(\begin{array}{c}
0.46 e^{0.53 \pi i}, \\
-0.36 e^{-0.20 \pi i}
\end{array}\right),\left(\begin{array}{c}
0.25 e^{0.29 \pi i}, \\
-0.16 e^{-0.18 \pi i}
\end{array}\right),\left(\begin{array}{c}
0.19 e^{0.11 \pi i}, \\
-0.11 e^{-0.34 \pi i}
\end{array}\right)\right)
$$

4.2.5. Conversational AI (CAI). The conversation is an essential part of human life, and it is one of the vital aspects of any business contact.

$$
\left(\operatorname{CAI},\left(\begin{array}{c}
0.43 e^{0.61 \pi i}, \\
-0.19 e^{-0.33 \pi i}
\end{array}\right),\left(\begin{array}{c}
0.27 e^{0.23 \pi i}, \\
-0.36 e^{-0.24 \pi i}
\end{array}\right),\left(\begin{array}{c}
0.17 e^{0.09 \pi i}, \\
-0.44 e^{-0.38 \pi i}
\end{array}\right)\right) .
$$

Then, the CBPFSs $G$ is;

$$
G=\left\{\begin{array}{l}
\left(M L,\left(\begin{array}{c}
0.45 e^{0.31 \pi i}, \\
-0.23 e^{-0.21 \pi i}
\end{array}\right),\left(\begin{array}{c}
0.28 e^{0.43 \pi i}, \\
-0.14 e^{-0.34 \pi i}
\end{array}\right),\left(\begin{array}{c}
0.27 e^{0.11 \pi i}, \\
-0.59 e^{-0.19 \pi i}
\end{array}\right)\right), \\
\left(N L P,\left(\begin{array}{c}
0.53 e^{0.41 \pi i}, \\
-0.34 e^{-0.11 \pi i}
\end{array}\right),\left(\begin{array}{c}
0.33 e^{0.21 \pi i}, \\
-0.24 e^{-0.24 \pi i}
\end{array}\right),\left(\begin{array}{c}
0.08 e^{0.21 \pi i}, \\
-0.40 e^{-0.17 \pi i}
\end{array}\right)\right), \\
\left(E S,\left(\begin{array}{c}
0.68 e^{0.51 \pi i}, \\
-0.44 e^{-0.01 \pi i}
\end{array}\right),\left(\begin{array}{c}
0.27 e^{0.29 \pi i}, \\
-0.23 e^{-0.14 \pi i}
\end{array}\right),\left(\begin{array}{c}
0.01 e^{0.16 \pi i}, \\
-0.29 e^{-0.20 \pi i}
\end{array}\right)\right), \\
\left(C V,\left(\begin{array}{c}
0.46 e^{0.53 \pi i}, \\
-0.36 e^{-0.20 \pi i}
\end{array}\right),\left(\begin{array}{c}
0.25 e^{0.29 \pi i}, \\
-0.16 e^{-0.18 \pi i}
\end{array}\right),\left(\begin{array}{c}
0.19 e^{0.11 \pi i}, \\
-0.11 e^{-0.34 \pi i}
\end{array}\right)\right), \\
\left(C A I,\left(\begin{array}{c}
0.43 e^{0.61 \pi i}, \\
-0.19 e^{-0.33 \pi i}
\end{array}\right),\left(\begin{array}{c}
0.27 e^{0.23 \pi i}, \\
-0.36 e^{-0.24 \pi i}
\end{array}\right),\left(\begin{array}{c}
0.17 e^{0.09 \pi i}, \\
-0.44 e^{-0.38 \pi i}
\end{array}\right)\right) .
\end{array}\right\} .
$$


4.3. Industries Using AI. Many industries take up AI technology to try to reduce operational costs, increase efficiency, grow revenue and improve customer experience. Almost every industry is utilizing the benefits of technology with the help of AI factors. Some industries are examined as follows. Table 2, represent the summary of industries.

4.3.1. Healthcare (HC). Artificial intelligence is already arriving as a game changer in the healthcare sector. Healthcare industries and research institutions are using NLP solutions and services to analyze large datasets. Furthermore, the increasing use of the Internet and connected devices, as well as huge amount of patient data during the spread of COVID19 , is expected to create numerous opportunities.

$$
\left(\operatorname{HC},\left(\begin{array}{c}
0.26 e^{0.65 \pi i}, \\
-0.22 e^{-0.31 \pi i}
\end{array}\right),\left(\begin{array}{c}
0.36 e^{0.14 \pi i}, \\
-0.35 e^{-0.24 \pi i}
\end{array}\right),\left(\begin{array}{c}
0.15 e^{0.21 \pi i}, \\
-0.39 e^{-0.23 \pi i}
\end{array}\right)\right) .
$$

4.3.2. Education (E). The classroom consists of AI-powered tactile robots for students that not only provide assistant with reading, learning the language and social interactions but also helps in developing vital social skills for children with disabilities.

$\left(\mathrm{E},\left(\begin{array}{c}0.39 e^{0.41 \pi i}, \\ -0.41 e^{-0.14 \pi i}\end{array}\right),\left(\begin{array}{c}0.15 e^{0.35 \pi i}, \\ -0.36 e^{-0.42 \pi i}\end{array}\right),\left(\begin{array}{c}0.34 e^{0.12 \pi i}, \\ -0.13 e^{-0.39 \pi i}\end{array}\right)\right)$.
TABLE 2: Summary of industries.

\begin{tabular}{lc}
\hline Industries & Abbreviation \\
\hline Healthcare & HC \\
Education & $\mathrm{E}$ \\
Agriculture & $\mathrm{A}$ \\
Cybersecurity & $\mathrm{CS}$ \\
\hline
\end{tabular}

4.3.3. Agriculture (A). AI systems are helping to improve the overall harvest and accuracy known as precision agriculture. AI technology helps in detecting disease in plants, pests and poor nutrition of farms. Expert systems were identified as a powerful tool with extensive potential in agriculture.

$\left(A,\left(\begin{array}{c}0.63 e^{0.59 \pi i}, \\ -0.38 e^{-0.23 \pi i}\end{array}\right),\left(\begin{array}{c}0.27 e^{0.26 \pi i}, \\ -0.29 e^{-0.39 \pi i}\end{array}\right),\left(\begin{array}{c}0.09 e^{0.13 \pi i}, \\ -0.30 e^{-0.19 \pi i}\end{array}\right)\right)$.

4.3.4. Cybersecurity (CS). AI can help discover devices and hidden patterns while processing large amount of data. AI is employed to protect the existing huge databases against virus attempts by wrongdoers who want to get access to some valuable or sensitive information.

$$
\left(C S,\left(\begin{array}{c}
0.34 e^{0.33 \pi i}, \\
-0.11 e^{-0.33 \pi i}
\end{array}\right),\left(\begin{array}{c}
0.23 e^{0.41 \pi i}, \\
-0.46 e^{-0.23 \pi i}
\end{array}\right),\left(\begin{array}{c}
0.18 e^{0.24 \pi i}, \\
-0.25 e^{-0.39 \pi i}
\end{array}\right)\right) .
$$

Then, the CBPFSs $F$ is;

$$
F=\left\{\begin{array}{l}
\left(H C,\left(\begin{array}{c}
0.26 e^{0.65 \pi i}, \\
-0.22 e^{-0.31 \pi i}
\end{array}\right),\left(\begin{array}{c}
0.36 e^{0.14 \pi i}, \\
-0.35 e^{-0.24 \pi i}
\end{array}\right),\left(\begin{array}{c}
0.15 e^{0.21 \pi i}, \\
-0.39 e^{-0.23 \pi i}
\end{array}\right)\right), \\
\left(E,\left(\begin{array}{c}
0.39 e^{0.41 \pi i}, \\
-0.41 e^{-0.14 \pi i}
\end{array}\right),\left(\begin{array}{c}
0.15 e^{0.35 \pi i}, \\
-0.36 e^{-0.42 \pi i}
\end{array}\right),\left(\begin{array}{c}
0.34 e^{0.12 \pi i}, \\
-0.13 e^{-0.39 \pi i}
\end{array}\right)\right), \\
\left(A,\left(\begin{array}{c}
0.63 e^{0.59 \pi i}, \\
-0.38 e^{-0.23 \pi i}
\end{array}\right),\left(\begin{array}{c}
0.27 e^{0.26 \pi i}, \\
-0.29 e^{-0.39 \pi i}
\end{array}\right),\left(\begin{array}{c}
0.09 e^{0.13 \pi i}, \\
-0.30 e^{-0.19 \pi i}
\end{array}\right)\right), \\
\left(C S,\left(\begin{array}{c}
0.34 e^{0.33 \pi i}, \\
-0.11 e^{-0.33 \pi i}
\end{array}\right),\left(\begin{array}{c}
0.23 e^{0.41 \pi i}, \\
-0.46 e^{-0.23 \pi i}
\end{array}\right),\left(\begin{array}{c}
0.18 e^{0.24 \pi i}, \\
-0.25 e^{-0.39 \pi i}
\end{array}\right)\right) .
\end{array}\right\}
$$

Now, determining the effects of every artificial intelligence factor on the industry, calculate the Cartesian product $G \times F$ in Table 3:

Each element of Cartesian product is an ordered pair which express the impact of AI factors on the industries. For example, $(\mathrm{ES}, A),\left(\begin{array}{c}0.63 e^{0.517 i} \\ -0.38 e^{-0.017 i}\end{array}\right),\left(\begin{array}{c}0.27 e^{0.26 \pi i} \\ -0.23 e^{-0.14 \pi i}\end{array}\right),\left(\begin{array}{c}0.09 e^{0.16 \pi i} \\ -0.30 e^{-0.20 \pi i}\end{array}\right)$ the agriculture industry is more improved using the expert system and in this ordered pair 0.63 explains the likeliness of effectiveness is good with high time duration of 0.51 , and -0.38 explains the unlikeness of effectiveness is also good with time duration of -0.01 . The 0.27 explains the likeliness of neutral effect with time duration 0.26 , and -0.23 explains the unlikeliness of neutral effect with time duration -0.14 . The 0.09 explains likeliness of ineffectiveness is low with time duration of 0.16 , and -0.30 explains unlikeliness of ineffectiveness is low with time duration of -0.20 . Hence, industries are more improved using the artificial intelligence. 
TABle 3: Cartesian product of $G \times F$.

\begin{tabular}{|c|c|c|c|}
\hline Ordered pair & Membership & Abstinence & Non-membership \\
\hline$(\mathrm{ML}, \mathrm{HC})$ & $\left(\begin{array}{c}0.26 e^{0.31 \pi i} \\
-0.22 e^{-0.21 \pi i}\end{array}\right)$ & $\left(\begin{array}{c}0.28 e^{0.14 \pi i} \\
-0.14 e^{-0.24 \pi i}\end{array}\right)$ & $\left(\begin{array}{c}0.27 e^{0.21 \pi i} \\
-0.59 e^{-0.23 \pi i}\end{array}\right)$ \\
\hline$(\mathrm{ML}, \mathrm{E})$ & $\left(\begin{array}{c}0.39 e^{0.31 \pi i} \\
-0.23 e^{-0.14 \pi i}\end{array}\right)$ & $\left(\begin{array}{c}0.15 e^{0.35 \pi i} \\
-0.14 e^{-0.34 \pi i}\end{array}\right)$ & $\left(\begin{array}{c}0.34 e^{0.12 \pi i} \\
-0.59 e^{-0.23 \pi i}\end{array}\right)$ \\
\hline$(\mathrm{ML}, \mathrm{A})$ & $\left(\begin{array}{c}0.45 e^{0.31 \pi i} \\
-0.23 e^{-0.21 \pi i}\end{array}\right)$ & $\left(\begin{array}{c}0.27 e^{0.26 \pi i} \\
-0.14 e^{-0.34 \pi i}\end{array}\right)$ & $\left(\begin{array}{c}0.27 e^{0.13 \pi i} \\
-0.59 e^{-0.19 \pi i}\end{array}\right)$ \\
\hline$(\mathrm{ML}, \mathrm{CS})$ & $\left(\begin{array}{c}0.34 e^{0.31 \pi i} \\
-0.11 e^{-0.21 \pi i}\end{array}\right)$ & $\left(\begin{array}{c}0.23 e^{0.41 \pi i} \\
-0.14 e^{-0.23 \pi i}\end{array}\right)$ & $\left(\begin{array}{c}0.27 e^{0.24 \pi i} \\
-0.59 e^{-0.39 \pi i}\end{array}\right.$ \\
\hline (NLP, HC) & $\left(\begin{array}{c}0.26 e^{0.41 \pi i} \\
-0.22 e^{-0.11 \pi i}\end{array}\right)$ & $\left(\begin{array}{c}0.33 e^{0.14 \pi i} \\
-0.24 e^{-0.24 \pi i}\end{array}\right)$ & $\left(\begin{array}{c}0.15 e^{0.21 \pi i} \\
-0.40 e^{-0.23 \pi i}\end{array}\right)$ \\
\hline$(\mathrm{NLP}, \mathrm{E})$ & $\left(\begin{array}{c}0.39 e^{0.41 \pi i} \\
-0.34 e^{-0.11 \pi i}\end{array}\right)$ & $\left(\begin{array}{c}0.15 e^{0.21 \pi i} \\
-0.24 e^{-0.24 \pi i}\end{array}\right)$ & $\left(\begin{array}{c}0.34 e^{0.21 \pi i} \\
-0.40 e^{-0.39 \pi i}\end{array}\right)$ \\
\hline$(\mathrm{NLP}, \mathrm{A})$ & $\left(\begin{array}{c}0.53 e^{0.41 \pi i} \\
-0.34 e^{-0.111 \pi i}\end{array}\right)$ & $\left(\begin{array}{c}0.27 e^{0.21 \pi i} \\
-0.24 e^{-0.24 \pi i}\end{array}\right)$ & $\left(\begin{array}{c}0.09 e^{0.21 \pi i} \\
-0.40 e^{-0.19 \pi i}\end{array}\right)$ \\
\hline (NLP, CS) & $\left(\begin{array}{c}0.34 e^{0.33 \pi i} \\
-0.11 e^{-0.11 \pi i}\end{array}\right)$ & $\left(\begin{array}{c}0.23 e^{0.21 \pi i} \\
-0.24 e^{-0.23 \pi i}\end{array}\right)$ & $\left(\begin{array}{c}0.18 e^{0.24 \pi i} \\
-0.40 e^{-0.39 \pi i}\end{array}\right)$ \\
\hline$(\mathrm{ES}, \mathrm{HC})$ & $\left(\begin{array}{c}0.26 e^{0.51 \pi i} \\
-0.22 e^{-0.01 \pi i}\end{array}\right)$ & $\left(\begin{array}{c}0.27 e^{0.14 \pi i} \\
-0.23 e^{-0.14 \pi i}\end{array}\right)$ & $\left(\begin{array}{c}0.15 e^{0.21 \pi i} \\
-0.39 e^{-0.23 \pi i}\end{array}\right)$ \\
\hline$(\mathrm{ES}, \mathrm{E})$ & $\left(\begin{array}{c}0.39 e^{0.41 \pi i} \\
-0.41 e^{-0.011 \pi i}\end{array}\right)$ & $\left(\begin{array}{c}0.15 e^{0.29 \pi i} \\
-0.23 e^{-0.14 \pi i}\end{array}\right)$ & $\left(\begin{array}{c}0.34 e^{0.16 \pi i} \\
-0.29 e^{-0.39 \pi i}\end{array}\right)$ \\
\hline$(\mathrm{ES}, \mathrm{A})$ & $\left(\begin{array}{c}0.63 e^{0.51 \pi i} \\
-0.38 e^{-0.011 \pi i}\end{array}\right)$ & $\left(\begin{array}{c}0.27 e^{0.26 \pi i} \\
-0.23 e^{-0.14 \pi i}\end{array}\right.$ & $\left(\begin{array}{c}0.09 e^{0.16 \pi i} \\
-0.30 e^{-0.20 \pi i}\end{array}\right.$ \\
\hline$(\mathrm{ES}, \mathrm{CS})$ & $\left(\begin{array}{c}0.34 e^{0.33 \pi i} \\
-0.11 e^{0.01 \pi i}\end{array}\right)$ & $\left(\begin{array}{c}0.23 e^{0.29 \pi i} \\
-0.23 e^{-0.14 \pi i}\end{array}\right.$ & $\left(\begin{array}{c}0.18 e^{0.24 \pi i} \\
-0.29 e^{-0.39 \pi i}\end{array}\right)$ \\
\hline$(\mathrm{CV}, \mathrm{HC})$ & $\left(\begin{array}{c}0.26 e^{0.53 \pi i} \\
-0.22 e^{-0.20 \pi i}\end{array}\right)$ & $\left(\begin{array}{c}0.25 e^{0.14 \pi i} \\
-0.16 e^{-0.18 \pi i}\end{array}\right.$ & $\left(\begin{array}{c}0.19 e^{0.21 \pi i} \\
-0.39 e^{-0.34 \pi i}\end{array}\right.$ \\
\hline$(\mathrm{CV}, \mathrm{E})$ & $\left(\begin{array}{c}0.39 e^{0.41 \pi i} \\
-0.36 e^{-0.14 \pi i}\end{array}\right)$ & $\left(\begin{array}{c}0.15 e^{0.29 \pi i} \\
-0.16 e^{-0.18 \pi i}\end{array}\right.$ & $\left(\begin{array}{c}0.34 e^{0.12 \pi i} \\
-0.13 e^{-0.39 \pi i}\end{array}\right)$ \\
\hline$(\mathrm{CV}, \mathrm{A})$ & $\left(\begin{array}{c}0.46 e^{0.53 \pi i} \\
-0.36 e^{-0.20 \pi i}\end{array}\right)$ & $\left(\begin{array}{c}0.25 e^{0.26 \pi i} \\
-0.16 e^{-0.18 \pi i}\end{array}\right.$ & $\left(\begin{array}{c}0.19 e^{0.13 \pi i} \\
-0.30 e^{-0.34 \pi i}\end{array}\right)$ \\
\hline$(\mathrm{CV}, \mathrm{CS})$ & $\left(\begin{array}{c}0.34 e^{0.33 \pi i} \\
-0.11 e^{-0.20 \pi i}\end{array}\right)$ & $\left(\begin{array}{c}0.23 e^{0.29 \pi i} \\
-0.16 e^{-0.18 \pi i}\end{array}\right.$ & $\left(\begin{array}{c}0.19 e^{0.24 \pi i} \\
-0.25 e^{-0.39 \pi i}\end{array}\right)$ \\
\hline (CAI, HC) & $\left(\begin{array}{c}0.26 e^{0.61 \pi i} \\
-0.19 e^{-0.31 \pi i}\end{array}\right)$ & $\left(\begin{array}{c}0.27 e^{0.14 \pi i} \\
-0.35 e^{-0.24 \pi i}\end{array}\right.$ & $\left(\begin{array}{c}0.17 e^{0.21 \pi i} \\
-0.44 e^{-0.38 \pi i}\end{array}\right)$ \\
\hline$(\mathrm{CAI}, \mathrm{E})$ & $\left(\begin{array}{c}0.39 e^{0.41 \pi i} \\
-0.19 e^{-0.14 \pi i}\end{array}\right)$ & $\left(\begin{array}{c}0.15 e^{0.23 \pi i} \\
-0.36 e^{-0.24 \pi i}\end{array}\right.$ & $\left(\begin{array}{c}0.34 e^{0.12 \pi i} \\
-0.44 e^{-0.39 \pi i}\end{array}\right)$ \\
\hline$(\mathrm{CAI}, \mathrm{A})$ & $\left(\begin{array}{c}0.43 e^{0.59 \pi i} \\
-0.19 e^{-0.23 \pi i}\end{array}\right)$ & $\left(\begin{array}{c}0.27 e^{0.23 \pi i} \\
-0.29 e^{-0.24 \pi i}\end{array}\right)$ & $\left(\begin{array}{c}0.17 e^{0.29 \pi i} \\
-0.44 e^{-0.38 \pi i}\end{array}\right)$ \\
\hline (CAI, CS) & $\left(\begin{array}{c}0.34 e^{0.33 \pi i} \\
-0.11 e^{-0.33 \pi i}\end{array}\right)$ & $\left(\begin{array}{c}0.23 e^{0.23 \pi i} \\
-0.36 e^{-0.23 \pi i}\end{array}\right)$ & $\left(\begin{array}{c}0.18 e^{0.24 \pi i} \\
-0.44 e^{-0.39 \pi i}\end{array}\right)$ \\
\hline
\end{tabular}

\section{Comparative Analysis}

This section explains the newly proposed concepts of CBPFR is more advanced by comparing with the pre-existing frameworks such as BFR, BIFR, BPFR, CBFR, CBIFR and BPFR.

5.1. BFR, BIFR, and BPFR with CBPFR. BFRs only explain the amplitude term of the membership degree. BIFRs only explain the amplitude term of the membership and non- membership degrees. Although, the BPFRs discuss the amplitude term of the membership, abstinence and non-membership degrees. But they can only deliberate the single variable with their simple structure. Whereas, the CBPFRs explain all of the three degrees with the ability to deal with the multidimensional variables due to their complex structure.

5.2. CBFR and CBIFR with CBPFR. Complex bipolar fuzzy relation discussing the membership degree and complex bipolar intuitionistic fuzzy relation explained the 
TABle 4: Cartesian product.

\begin{tabular}{|c|c|c|}
\hline Ordered pair & Membership & Non-membership \\
\hline$(\mathrm{ML}, \mathrm{HC})$ & $\left(\begin{array}{c}0.26 e^{0.31 \pi i} \\
-0.22 e^{-0.21 \pi i}\end{array}\right)$ & $\left(\begin{array}{c}0.27 e^{0.21 \pi i} \\
-0.59 e^{-0.23 \pi i}\end{array}\right.$ \\
\hline$(\mathrm{ML}, \mathrm{E})$ & $\left(\begin{array}{c}0.39 e^{0.31 \pi i} \\
-0.23 e^{-0.14 \pi i}\end{array}\right)$ & $\left(\begin{array}{c}0.34 e^{0.12 \pi i} \\
-0.59 e^{-0.23 \pi i}\end{array}\right.$ \\
\hline$(\mathrm{ML}, \mathrm{A})$ & $\left(\begin{array}{c}0.45 e^{0.31 \pi i} \\
-0.23 e^{-0.21 \pi i}\end{array}\right)$ & $\left(\begin{array}{c}0.27 e^{0.13 \pi i} \\
-0.59 e^{-0.19 \pi i}\end{array}\right.$ \\
\hline$(\mathrm{ML}, \mathrm{CS})$ & $\left(\begin{array}{c}0.34 e^{0.31 \pi i} \\
-0.11 e^{-0.21 \pi i}\end{array}\right)$ & $\left(\begin{array}{c}0.27 e^{0.24 \pi i} \\
-0.59 e^{-0.39 \pi i}\end{array}\right.$ \\
\hline (NLP, HC) & $\left(\begin{array}{c}0.26 e^{0.41 \pi i} \\
-0.22 e^{-0.11 \pi i}\end{array}\right)$ & $\left(\begin{array}{c}0.15 e^{0.21 \pi i} \\
-0.40 e^{-0.23 \pi i}\end{array}\right.$ \\
\hline$(\mathrm{NLP}, \mathrm{E})$ & $\left(\begin{array}{c}0.39 e^{0.41 \pi i} \\
-0.34 e^{-0.11 \pi i}\end{array}\right)$ & $\left(\begin{array}{c}0.34 e^{0.21 \pi i} \\
-0.40 e^{-0.39 \pi i}\end{array}\right.$ \\
\hline$(\mathrm{NLP}, \mathrm{A})$ & $\left(\begin{array}{c}0.53 e^{0.41 \pi i} \\
-0.34 e^{-0.11 \pi i}\end{array}\right)$ & $\left(\begin{array}{c}0.09 e^{0.21 \pi i} \\
-0.40 e^{-0.19 \pi i}\end{array}\right.$ \\
\hline (NLP, CS) & $\left(\begin{array}{c}0.34 e^{0.33 \pi i} \\
-0.11 e^{-0.11 \pi i}\end{array}\right)$ & $\left(\begin{array}{c}0.18 e^{0.24 \pi i} \\
-0.40 e^{-0.39 \pi i}\end{array}\right.$ \\
\hline$(\mathrm{ES}, \mathrm{HC})$ & $\left(\begin{array}{c}0.26 e^{0.51 \pi i} \\
-0.22 e^{-0.01 \pi i}\end{array}\right)$ & $\left(\begin{array}{c}0.15 e^{0.21 \pi i} \\
-0.39 e^{-0.23 \pi i}\end{array}\right.$ \\
\hline$(\mathrm{ES}, \mathrm{E})$ & $\left(\begin{array}{c}0.39 e^{0.41 \pi i} \\
-0.41 e^{-0.01 \pi i}\end{array}\right)$ & $\left(\begin{array}{c}0.34 e^{0.16 \pi i} \\
-0.29 e^{-0.39 \pi i}\end{array}\right.$ \\
\hline$(\mathrm{ES}, \mathrm{A})$ & $\left(\begin{array}{c}0.63 e^{0.51 \pi i} \\
-0.38 e^{-0.01 \pi i}\end{array}\right)$ & $\left(\begin{array}{c}0.09 e^{0.16 \pi i} \\
-0.30 e^{-0.20 \pi i}\end{array}\right.$ \\
\hline$(\mathrm{ES}, \mathrm{CS})$ & $\left(\begin{array}{c}0.34 e^{0.33 \pi i} \\
-0.11 e^{0.01 \pi i}\end{array}\right)$ & $\left(\begin{array}{c}0.18 e^{0.24 \pi i} \\
-0.29 e^{-0.39 \pi i}\end{array}\right.$ \\
\hline$(\mathrm{CV}, \mathrm{HC})$ & $\left(\begin{array}{c}0.26 e^{0.53 \pi i} \\
-0.22 e^{-0.20 \pi i}\end{array}\right)$ & $\left(\begin{array}{c}0.19 e^{0.21 \pi i} \\
-0.39 e^{-0.34 \pi i}\end{array}\right.$ \\
\hline$(\mathrm{CV}, \mathrm{E})$ & $\left(\begin{array}{c}0.39 e^{0.41 \pi i} \\
-0.36 e^{-0.14 \pi i}\end{array}\right)$ & $\left(\begin{array}{c}0.34 e^{0.12 \pi i} \\
-0.13 e^{-0.39 \pi i}\end{array}\right.$ \\
\hline$(\mathrm{CV}, \mathrm{A})$ & $\left(\begin{array}{c}0.46 e^{0.53 \pi i} \\
-0.36 e^{-0.20 \pi i}\end{array}\right)$ & $\left(\begin{array}{c}0.19 e^{0.13 \pi i} \\
-0.30 e^{-0.34 \pi i}\end{array}\right.$ \\
\hline$(\mathrm{CV}, \mathrm{CS})$ & $\left(\begin{array}{c}0.34 e^{0.33 \pi i} \\
-0.11 e^{-0.20 \pi i}\end{array}\right)$ & $\left(\begin{array}{c}0.19 e^{0.24 \pi i} \\
-0.25 e^{-0.39 \pi i}\end{array}\right.$ \\
\hline (CAI, HC) & $\left(\begin{array}{c}0.26 e^{0.61 \pi i} \\
-0.19 e^{-0.31 \pi i}\end{array}\right)$ & $\left(\begin{array}{c}0.17 e^{0.21 \pi i} \\
-0.44 e^{-0.38 \pi i}\end{array}\right.$ \\
\hline (CAI, E) & $\left(\begin{array}{c}0.39 e^{0.41 \pi i} \\
-0.19 e^{-0.14 \pi i}\end{array}\right)$ & $\left(\begin{array}{c}0.34 e^{0.12 \pi i} \\
-0.44 e^{-0.39 \pi i}\end{array}\right.$ \\
\hline$(\mathrm{CAI}, \mathrm{A})$ & $\left(\begin{array}{c}0.43 e^{0.59 \pi i} \\
-0.19 e^{-0.23 \pi i}\end{array}\right)$ & $\left(\begin{array}{c}0.17 e^{0.29 \pi i} \\
-0.44 e^{-0.38 \pi i}\end{array}\right.$ \\
\hline (CAI, CS) & $\left(\begin{array}{c}0.34 e^{0.33 \pi i} \\
-0.11 e^{-0.33 \pi i}\end{array}\right)$ & $\left(\begin{array}{c}0.18 e^{0.24 \pi i} \\
-0.44 e^{-0.39 \pi i}\end{array}\right.$ \\
\hline
\end{tabular}

TABle 5: Summary of comparative analysis.

\begin{tabular}{lcccc}
\hline Structure & Membership & Abstinence & Non-membership & Multi-dimension \\
\hline BFS & Yes & No & No & No \\
CBFS & Yes & No & No & Yes \\
BIFS & Yes & No & Yes & No \\
CBIFS & Yes & No & Yes & Yes \\
BPFS & Yes & Yes & Yes & No \\
CBPFS & Yes & Yes & & Yes \\
\hline
\end{tabular}


membership and non-membership degree with multivariable. These structures are able to solve the multi-dimensional problems. On the other hand, CBPFR also explained the neutral effect of one object to the other. It is the wide range form as compared to CBFR and CBIFR. Here, take the following two sets $G$ and $F$ of previous example with CBIFRs respectively.

$$
\begin{aligned}
& G=\left\{\begin{array}{l}
\left(\operatorname{ML},\left(\begin{array}{c}
0.45 e^{0.31 \pi i}, \\
-0.23 e^{-0.21 \pi i}
\end{array}\right),\left(\begin{array}{c}
0.27 e^{0.51 \pi i}, \\
-0.59 e^{-0.19 \pi i}
\end{array}\right)\right), \\
\left(\operatorname{NLP},\left(\begin{array}{c}
0.53 e^{0.41 \pi i}, \\
-0.34 e^{-0.11 \pi i}
\end{array}\right),\left(\begin{array}{c}
0.24 e^{0.21 \pi i}, \\
-0.48 e^{-0.17 \pi i}
\end{array}\right)\right), \\
\left(\operatorname{ES},\left(\begin{array}{c}
0.78 e^{0.51 \pi i}, \\
-0.44 e^{-0.01 \pi i}
\end{array}\right),\left(\begin{array}{c}
0.11 e^{0.16 \pi i}, \\
-0.29 e^{-0.20 \pi i}
\end{array}\right)\right), \\
\left(\operatorname{CV},\left(\begin{array}{c}
0.46 e^{0.53 \pi i}, \\
-0.56 e^{-0.20 \pi i}
\end{array}\right),\left(\begin{array}{c}
0.34 e^{0.31 \pi i}, \\
-0.11 e^{-0.34 \pi i}
\end{array}\right)\right), \\
\left(\operatorname{CAI},\left(\begin{array}{c}
0.43 e^{0.61 \pi i}, \\
-0.19 e^{-0.33 \pi i}
\end{array}\right),\left(\begin{array}{c}
0.17 e^{0.29 \pi i}, \\
-0.44 e^{-0.38 \pi i}
\end{array}\right)\right),
\end{array}\right\} \\
& F=\left\{\begin{array}{c}
\left(H C,\left(\begin{array}{c}
0.26 e^{0.67 \pi i}, \\
-0.52 e^{-0.31 \pi i}
\end{array}\right),\left(\begin{array}{c}
0.51 e^{0.21 \pi i}, \\
-0.39 e^{-0.23 \pi i}
\end{array}\right)\right), \\
\left(E,\left(\begin{array}{c}
0.39 e^{0.41 \pi i}, \\
-0.41 e^{-0.34 \pi i}
\end{array}\right),\left(\begin{array}{c}
0.64 e^{0.42 \pi i}, \\
-0.13 e^{-0.39 \pi i}
\end{array}\right)\right), \\
\left(A,\left(\begin{array}{c}
0.73 e^{0.59 \pi i}, \\
-0.38 e^{-0.23 \pi i}
\end{array}\right),\left(\begin{array}{c}
0.14 e^{0.25 \pi i}, \\
-0.39 e^{-0.19 \pi i}
\end{array}\right)\right), \\
\left(C S,\left(\begin{array}{c}
0.34 e^{0.33 \pi i}, \\
-0.11 e^{-0.33 \pi i}
\end{array}\right),\left(\begin{array}{c}
0.49 e^{0.44 \pi i}, \\
-0.25 e^{-0.39 \pi i}
\end{array}\right)\right) .
\end{array}\right\} .
\end{aligned}
$$

Then, their Cartesian product explained in Table 4.

Each ordered pair of $F \times G$ is defined the effectiveness and ineffectiveness of industries to artificial intelligence factors. They do not describe the neutral effect. These structures are providing the limited information. But CBPFR are produce the better results with the detailed information. Table 5, explain the summary of comparative analysis.

\section{Conclusion}

In this paper, we defined the newly proposed concepts of complex bipolar picture fuzzy set (CBPFS). Moreover, the discussed the complex bipolar picture fuzzy relation (CBPFR), investigating the Cartesian product of two CBPFSs. Additionally, some types of CBPFRs are examined such as CBP reflexive FR, CBP irreflexive FR, CBP complete $\mathrm{FR}, \mathrm{CBP}$ antisymmetric FR, CBP transitive FR, CBP equivalence FR, CBP linear order FR, CBP strict FR and many more. The proposed study defines the relationship industries to make the better by using the artificial intelligence factor. The CBPFR are the more improved form of all pre-defined structures such as FS, CFS, IFS, CIFS, PFS,
CPFS, BFS, CBFS, BIFS, CBIFS, and CBPFS. The advantage of the proposed structures is that they discuss all of the three features i.e., membership, abstinence and non-membership with multi-dimensions. Moreover, the CBPFR also talk about the positive and negative effects simultaneously. In addition, these notions include the phase terms, which enables thm to model periodicity. An interesting and novel application of the proposed framework was presented which investigated the effects in different industries due to AI technologies. Furthermore, a detailed comparative analysis was carried out to verify the effectiveness and strength of proposed methods. In future, we would be looking forward to extend these ideas which can be applied to a vast range of fields such as economics, statistics, business, and sciences of computer, medical, agriculture, chemicals, matter, and others.

\section{Data Availability}

No data set was generated or analyzed during the current study.

\section{Conflicts of Interest}

The authors do not have any sorts of conflicts of interest.

\section{Acknowledgments}

This research was supported by the Researchers Supporting Project number (RSP2022R476), King Saud University, Riyadh, Saudi Arabia.

\section{References}

[1] L. A. Zadeh, "Fuzzy sets," Information and Control, vol. 8, no. 3, pp. 338-353, 1965.

[2] G. J. Klir and T. A. Folger, Fuzzy Sets, Uncertainty, and Information, Prentice-Hall, Hoboken, NJ, USA, 1988.

[3] C. V. Negoiţă and D. A. Ralescu, Applications of Fuzzy Sets to Systems Analysis, Birkhäuser, Basel, Switzerland, 1975.

[4] S. Laengle, V. Lobos, J. M. Merigó, E. Herrera-Viedma, M. J. Cobo, and B. De Baets, "Forty years of fuzzy sets and systems: a bibliometric analysis," Fuzzy Sets and Systems, vol. 402, pp. 155-183, 2021.

[5] W.-J. Xu, L.-J. He, and G.-Y. Zhu, "Many-objective flow shop scheduling optimisation with genetic algorithm based on fuzzy sets," International Journal of Production Research, vol. 59, no. 3, pp. 702-726, 2021.

[6] S. Mewada, P. Sharma, and S. S. Gautam, "Exploration of fuzzy system with applications," in Research Anthology on Multi-Industry Uses of Genetic Programming and Algorithms, pp. 550-569, IGI Global, New York, NY, USA, 2021.

[7] K.-P. Adlassnig, "Fuzzy set theory in medical diagnosis," IEEE Transactions on Systems, Man, and Cybernetics, vol. 16, no. 2, pp. 260-265, 1986.

[8] J. Lu and D. Ruan, Multi-objective Group Decision Making: Methods, Software and Applications with Fuzzy Set Techniques, Vol. 6, Imperial College Press, , London, UK, 2007.

[9] M. Gehrke, C. Walker, and E. Walker, "Some comments on interval valued fuzzy sets," Structure, vol. 1, p. 2, 1996.

[10] J. M. Mendel, "Fuzzy logic systems for engineering: a tutorial," Proceedings of the IEEE, vol. 83, no. 3, pp. 345-377, 1995. 
[11] D. Yu, Q. Hu, and C. Wu, "Uncertainty measures for fuzzy relations and their applications," Applied Soft Computing, vol. 7, no. 3, pp. 1135-1143, 2007.

[12] R. T. Yeh and S. Y. Bang, "Fuzzy relations, fuzzy graphs, and their applications to clustering analysis," in Fuzzy Sets and Their Applications to Cognitive and Decision Processes, pp. 125-149, Academic Press, Cambridge, MA, USA, 1975.

[13] M. Elkano, J. A. Sanz, M. Galar, B. Pekala, U. Bentkowska, and H. Bustince, "Composition of interval-valued fuzzy relations using aggregation functions," Information Sciences, vol. 369, pp. 690-703, 2016.

[14] K. T. Atanassov, Intuitionistic Fuzzy Sets, Physica, Heidelberg, Germany, 1999.

[15] E. Szmidt and J. Kacprzyk, "Distances between intuitionistic fuzzy sets," Fuzzy Sets and Systems, vol. 114, no. 3, pp. $505-518,2000$.

[16] S. K. De, R. Biswas, and A. R. Roy, "Some operations on intuitionistic fuzzy sets," Fuzzy Sets and Systems, vol. 114, no. 3, pp. 477-484, 2000.

[17] P. Burillo and H. Bustince, "Intuitionistic fuzzy relations (Part I)," Mathware and Soft Computing, vol. 2, no. 1, pp. 5-38, 1995.

[18] G. Deschrijver and E. E. Kerre, "On the composition of intuitionistic fuzzy relations," Fuzzy Sets and Systems, vol. 136, no. 3, pp. 333-361, 2003.

[19] B. C. Cuong and V. Kreinovich, "Picture Fuzzy Sets-a new concept for computational intelligence problems," in Proceedings of the 3rd World Congress On Information and Communication Technologies (WICT 2013), pp. 1-6, IEEE, Hanoi, Vietnam, 2013 December.

[20] A. H. Ganie, S. Singh, and P. K. Bhatia, "Some new correlation coefficients of picture fuzzy sets with applications," Neural Computing of Applications, pp. 1-17, 2020.

[21] P. Liu, M. Munir, T. Mahmood, and K. Ullah, "Some similarity measures for interval-valued picture fuzzy sets and their applications in decision making," Information, vol. 10, no. 12, p. $369,2019$.

[22] D. Ramot, R. Milo, M. Friedman, and A. Kandel, "Complex fuzzy sets," IEEE Transactions on Fuzzy Systems, vol. 10, no. 2, pp. 171-186, 2002.

[23] D. E. Tamir, N. D. Rishe, and A. Kandel, "Complex fuzzy sets and complex fuzzy logic an overview of theory and applications," Fifty years of fuzzy logic and its applications, vol. 326, pp. 661-681, 2015.

[24] S. Greenfield, F. Chiclana, and S. Dick, "Interval-valued complex fuzzy logic," in In Proceedings of the 2016 IEEE International Conference on Fuzzy Systems (FUZZ-IEEE), pp. 2014-2019, IEEE, Vancouver, BC, Canada, July 2016.

[25] A. Nasir, N. Jan, A. Gumaei, and S. U. Khan, "Medical diagnosis and life span of sufferer using interval valued complex fuzzy relations," IEEE Access, vol. 9, pp. 93764-93780, 2021.

[26] J. Ma, G. Zhang, and J. Lu, "A method for multiple periodic factor prediction problems using complex fuzzy sets," IEEE Transactions on Fuzzy Systems, vol. 20, no. 1, pp. 32-45, 2011.

[27] A. M. D. J. S. Alkouri and A. R. Salleh, "Complex intuitionistic fuzzy sets," in Proceedings of the International Conference On Fundamental And Applied Sciences 2012, vol. 1482, no. 1, pp. 464-470, Kuala Lumpur, Malaysia, September 2012.

[28] H. Garg and D. Rani, "Some results on information measures for complex intuitionistic fuzzy sets," International Journal of Intelligent Systems, vol. 34, no. 10, pp. 2319-2363, 2019.

[29] N. Jan, A. Nasir, M. Alhilal, S. Khan, D. Pamucar, and A. Alothaim, "Investigation of cyber-security and cybercrimes in oil and gas sectors using the innovative structures of complex intuitionistic fuzzy relations," Entropy, vol. 23, no. 9, p. 1112, 2021.

[30] A. Nasir, N. Jan, A. Gumaei, S. U. Khan, and F. R. Albogamy, "Cybersecurity against the loopholes in industrial control systems using interval-valued complex intuitionistic fuzzy relations," Applied Sciences, vol. 11, no. 16, p. 7668, 2021.

[31] M. Akram, A. Bashir, and H. Garg, "Decision-making model under complex picture fuzzy Hamacher aggregation operators," Computational and Applied Mathematics, vol. 39, no. 3, pp. 1-38, 2020.

[32] A. Nasir, N. Jan, S. U. Khan, A. Gumaei, and A. Alothaim, "analysis of communication and network securities using the concepts of complex picture fuzzy relations," Computational Intelligence and Neuroscience, vol. 2021, Article ID 9427492, 20 pages, 2021.

[33] W. R. Zhang, "Bipolar fuzzy sets and relations: a computational framework for cognitive modeling and multiagent decision analysis," in Proceedings of the NAFIPS/IFIS/ NASA'94 1st International Joint Conference of the North American Fuzzy Information Processing Society Biannual Conference. The Industrial Fuzzy Control and Intelligence, pp. 305-309, IEEE, San Antonio, TX, USA, December 1994.

[34] K. M. Lee, "Bipolar-valued fuzzy sets and their operations," in Proceedings of the International Conference on Intelligent Technologies, pp. 307-312, Bangkok, Thailand, December 2000.

[35] T. Mahmood and K. Hayat, "Characterizations of hemi-rings by their bipolar-valued fuzzy h-ideals," Information Sciences Letters, vol. 4, no. 2, p. 1, 2015.

[36] M. Akram, S. Samanta, and M. Pal, "Application of bipolar fuzzy sets in planar graphs," International Journal of Algorithms, Computing and Mathematics, vol. 3, no. 2, pp. 773785, 2017.

[37] A. U. M. J. Alkouri, M. O. Massa'deh, and M. Ali, "On bipolar complex fuzzy sets and its application," Journal of Intelligent and Fuzzy Systems, vol. 39, no. 1, pp. 383-397, 2020.

[38] D. Ezhilmaran and K. Sankar, "Morphism of bipolar intuitionistic fuzzy graphs," Journal of Discrete Mathematical Sciences and Cryptography, vol. 18, no. 5, pp. 605-621, 2015.

[39] M. S. Sindhu, T. Rashid, and A. Kashif, "An approach to select the investment based on bipolar picture fuzzy sets," International Journal of Fuzzy Systems, pp. 1-13, 2021.

[40] N. J. Nilsson, Principles of Artificial Intelligence, Springer Science \& Business Media, Heidelberg, Germany, 1982.

[41] A. Ramesh, C. Kambhampati, J. Monson, and P. Drew, "Artificial intelligence in medicine," Annals of the Royal College of Surgeons of England, vol. 86, no. 5, pp. 334-338, 2004.

[42] T. W. Rondeau, "Application of artificial intelligence to wireless communications," Doctoral Dissertation, Virginia Tech, Blacksburg, VA, USA, 2007.

[43] T. Hirasawa, K. Aoyama, T. Tanimoto et al., "Application of artificial intelligence using a convolutional neural network for detecting gastric cancer in endoscopic images," Gastric Cancer, vol. 21, no. 4, pp. 653-660, 2018.

[44] N. Anantrasirichai and D. Bull, "Artificial intelligence in the creative industries: a review," Artificial Intelligence Review, vol. 55, pp. 1-68, 2021.

[45] K.-H. Yu, A. L. Beam, and I. S. Kohane, "Artificial intelligence in healthcare," Nature biomedical engineering, vol. 2, no. 10, pp. 719-731, 2018. 